\title{
ULF pulsations observed by the ETS-VI satellite: Substorm associated azimuthal Pc 4 pulsations on the nightside
}

\author{
M. Nosé ${ }^{1}$, T. Iyemori ${ }^{1}$, S. Nakabe ${ }^{1}$, T. Nagai ${ }^{2}$, H. Matsumoto ${ }^{3}$, and T. Goka ${ }^{3}$ \\ ${ }^{1}$ Faculty of Science, Kyoto University, Kyoto 606-8502, Japan \\ ${ }^{2}$ Earth and Planetary Sciences, Tokyo Institute of Technology, Tokyo 152-8551, Japan \\ ${ }^{3}$ Office of Research and Development, National Space Development Agency of Japan, Tsukuba 305-0047, Japan
}

(Received July 1, 1997; Revised October 6, 1997; Accepted October 29, 1997)

\begin{abstract}
The magnetic field data from the Engineering Test Satellite -VI (ETS-VI) have been analyzed to investigate the occurrence distributions of pulsations in Pc 3-5 frequency ranges in the magnetosphere. The observation of ETS-VI covered the invariant latitude (ILAT) range of $64.5^{\circ}-69^{\circ} \mathrm{ILAT}$ near the geomagnetic equator $\left(-10^{\circ}-20^{\circ}\right.$ magnetic latitude) at all magnetic local time (MLT). Magnetic pulsations were selected by the Fast Fourier Transform method and checked by visual scanning if they have continuous waveforms. From the occurrence distributions of pulsations, we have found distinctive features in the following pulsations: (1) azimuthal Pc 5 pulsation; (2) azimuthal Pc 3 pulsation; (3) radial Pc 4 pulsation on the dayside; (4) azimuthal Pc 4 pulsations on the nightside. In respect of the first three types of pulsations (i.e., the azimuthal Pc 5 pulsation, the azimuthal Pc 3 pulsation, and the radial Pc 4 pulsation on the dayside), the results presented in this study confirm the previous results obtained by other satellite observations. The azimuthal Pc 4 pulsations on the nightside were observed in continuous waveforms lasting for about 10 minutes. Although the azimuthal Pc 4 pulsations on the nightside start at almost the same time as substorm onsets, they are different from $\mathrm{Pi} 2$ pulsations in the magnetosphere. They are observed frequently in the MLT range of 23-04MLT with an occurrence peak at 01-02MLT. We suggest that the azimuthal Pc 4 pulsations on the nightside are excited through coupling to the fast mode Alfvén waves which were launched at substorm onset.
\end{abstract}

\section{Introduction}

Geomagnetic pulsations which have continuous waveforms are called Pc-type pulsations. The Pc-type pulsations are classified into 6 main classes according to their period. (Jacobs et al., 1964; Saito, 1978). The pulsations with period ranges of 10-45 seconds, 45-150 seconds, and 150-600 seconds are denoted as Pc 3, Pc 4, and Pc 5 pulsations, respectively. Pc 3-5 pulsations observed by satellites have been investigated by many researchers since the 1970s. A brief review of the previous studies employing satellite data which were published before 1989 is found in Anderson et al. (1990a).

Arthur and McPherron (1977) studied statistically 81 events of transverse Pc 3 pulsations observed by ATS-1, which were simultaneously observed on the ground. The transverse Pc 3 pulsations had the local time distribution with a strong midmorning peak and dominant frequencies between $40 \mathrm{mHz}$ and $50 \mathrm{mHz}$. From analysis of the magnetic field data obtained by ATS-6, Arthur and McPherron (1981) found that radially polarized waves in the Pc 4 ranges occur predominantly at dusk.

Using the magnetic field data from the geosynchronous satellites, Takahashi and McPherron (1984) studied two different types of waves, that is, azimuthally polarized waves involving the fundamental and several harmonics, and radially polarized waves excited at the second harmonic. The former were observed through the dayside, and the occur-

Copy right(C) The Society of Geomagnetism and Earth, Planetary and Space Sciences (SGEPSS); The Seismological Society of Japan; The Volcanological Society of Japan; The Geodetic Society of Japan; The Japanese Society for Planetary Sciences. rence of the fundamental wave was more biased toward the morning than that of harmonic waves. The latter were observed mainly on the afternoon side. They concluded that the azimuthal waves with fundamental and harmonics are driven externally, and the radial waves at second harmonic are likely to be excited internally through wave-particle interactions.

Kokubun et al. (1989) studied R-class (radial) Pc 4 waves and A-class (azimuthal) Pc 4-5 waves with the magnetic field data and particle flux data from ATS-6. Their results showed that R-class Pc 4 waves predominantly occur in the afternoon sector from 12 to $18 \mathrm{LT}$, and A-class Pc 4-5 waves with the period range of 100-200 seconds predominantly appear in the morning sector. The oscillation of the azimuthal component for A-class Pc 4-5 waves was out of phase with proton flux $(<120 \mathrm{keV})$, though the phase relationship for R-class Pc 4 waves was dependent on both energy and pitch angle of proton flux.

ULF pulsations ( $f=0-80 \mathrm{mHz}$ ) observed by the AMPTE/CCE satellite from $L=5$ to 9 in the equatorial magnetosphere were statistically investigated by Anderson et al. (1990a). The occurrence distribution of fundamental toroidal waves in Pc 5 band had a dawn/dusk asymmetry, that is, the waves were observed frequently for $L=8-9$ at dawn but were less observed at dusk. They found that harmonic toroidal waves in Pc 3 band were dominant on the dayside, particularly in the prenoon hours. The toroidal Pc 3 waves occurred with frequencies which varied continuously with radial distance. Radially polarized pulsations in the Pc 4 frequency range were found to occur in width extending from 11 magnetic local time (MLT) through dusk to 04MLT. The 
radial Pc 4 pulsations were frequently observed for $L<7$ at noon and for $L>7$ on the nightside.

Takahashi and Anderson (1992) examined spatial distribution of ULF ( $f<80 \mathrm{mHz}$ ) energy in the inner magnetosphere $(L=2.5-6.5)$, using the magnetic field data from the AMPTE/CCE satellite. They found that multiharmonic toroidal oscillations in the Pc 3 and Pc 4 frequency ranges appeared predominantly on the dayside. Assuming that the toroidal oscillations are due to standing Alfvén waves, they determined the radial profile of the plasma mass density and Alfvén wave velocity.

Using the data from the AMPTE/CCE satellite and geosynchronous GOES-5 and -6 satellites, Takahashi et al. (1996) reported toroidal magnetic pulsations in the nightside magnetosphere in the form of transient oscillations typically lasting 10-20 minutes. These waves were called as transient toroidal waves (TTW). They showed two examples of TTW with frequency ranges of $11-17 \mathrm{mHz}$ for $L=5.5$ to 7 and one example of TTW which consisted of a few discrete spectral components $(5,10,16$, and $28 \mathrm{mHz})$, and concluded that TTW are standing waves. It was also found that TTW on the nightside for $4<L<7$ were observed immediately following $\sim 30 \%$ of substorm onsets.

Saka et al. (1996a) found that quasi-periodic oscillations (QPO) with frequency of $9 \mathrm{mHz}$ were observed by GOES 5 and 6 in the night sector in association with the onset of ground Pi 2 pulsations. They also found that $16 \%$ of Pi 2 events ( 35 of 216 Pi 2 events) were accompanied by QPO. The QPO were polarized more or less in the azimuthal direction. The QPO in the postmidnight sector had more sinusoidal waveforms than those in the premidnight, and had an occurrence peak at 02MLT.

In this paper we aim to investigate characteristics of pulsations in Pc 3-5 bands observed by Engineering Test SatelliteVI (ETS-VI). In respect of the azimuthal Pc 5 pulsations, the azimuthal Pc 3 pulsations, and the radial Pc 4 pulsations on the dayside, the results were consistent with those by the previous studies. We examined statistically the azimuthal Pc 4 pulsations on the nightside, which are considered as the same phenomena as TTW and QPO, and concluded that the azimuthal $\mathrm{Pc} 4$ pulsations on the nightside are excited through coupling to the fast mode Alfvén waves which are radiated at substorm onsets. This excitation mechanism is different from that by the previous studies. The data used here and a procedure to select pulsations will be introduced in Section 2. Waveforms and occurrence distributions for the 4 kinds of pulsations are presented in Section 3. In Section 4, the results will be discussed and compared with the previous statistical studies.

\section{Experiment and Data Analysis}

\subsection{The satellite and magnetometer}

The ETS-VI satellite, which was launched on August 28, 1994 , had a perigee of $2.3 R_{E}$, an apogee of $7.1 R_{E}$, an inclination of $13.4^{\circ}$, and a 14.4 -hour orbital period. The triaxial fluxgate magnetometer (MAM) on board ETS-VI has two observation modes, Range-L mode and Range-H mode. The dynamic range and resolution in Range- $\mathrm{L}$ mode are $\pm 65536 \mathrm{nT}$ and $32 \mathrm{nT}$, respectively. Range- $\mathrm{H}$ mode has the dynamic range of $\pm 256 \mathrm{nT}$ and the resolution of $0.125 \mathrm{nT}$.
Range-H is used over approximately 11 hours at radial distances of $5.0-7.1 R_{E}$ and $-15^{\circ}$ to $+25^{\circ}$ magnetic latitudes (MLAT), and Range-L is used near perigee. The data were recorded with a sampling interval of 3 seconds. A more detailed description of the ETS-VI satellite and its observations are found in Nagai et al. (1996).

\subsection{Data coverage}

The data period used here is 496 days in total, from October 1994 through April 1996. We excluded the data period when the satellite was not three-axis stabilized. Only the data obtained near the apogee (where the altitude of the ETS-VI satellite is more than $28000 \mathrm{~km}$ ) were used in this study, because the data obtained with Range-L mode are not useful for pulsation studies in respect of their low resolution. The ETS-VI satellite has changed the local time of the apogee from about 05MLT in November 1994, through midnight, to about 17MLT in June 1995, and again to about 05MLT in December 1995. The local time of the apogee in April 1996 was about 20MLT. Therefore the data set used here covers all MLT ranges, although the number of the data points on the nightside (20-05MLT) is larger than that on the dayside. Plates 1(a) and 1(b) give the distributions of data set in MLT-MLAT plane and MLT-invariant latitude (ILAT) plane, respectively. One mesh in Plate 1(a) corresponds to $1^{\circ}$ of MLAT by 1 hour of MLT, and in Plate $1(b), 0.5^{\circ}$ of ILAT by 1 hour of MLT. The color images show hours of observation time. From Plate 1(a) we notice that generally the higher latitudes than $20^{\circ}$ MLAT and $-10^{\circ}$ MLAT are less or not completely covered. The observation time in MLT-MLAT areas of $04-10 \mathrm{MLT},-5^{\circ}$ to $-10^{\circ}$ MLAT and $10-17 \mathrm{MLT}, 7^{\circ}-12^{\circ} \mathrm{MLAT}$ are small. The coverage in ILAT is biased around $67^{\circ}-68^{\circ}$ ILAT (Plate $1(\mathrm{~b})$ ), because the ETSVI satellite with the small inclination stayed for a long time near the apogee of 7.1 $R_{E}$. Observation time in the ILAT ranges higher than $69^{\circ}$ ILAT and lower than $64.5^{\circ}$ ILAT are small at all MLT.

\subsection{Preparation of pulsation list}

We adopted the following procedures to make the lists of Pc-type geomagnetic pulsations. In the first step we have made the magnetic field variation data by subtracting the IGRF90 model fields from the observed magnetic fields. The magnetic field variation data were represented in the local magnetic (LMG) coordinate system, in which the $\Delta B_{\|}$component is the tangential direction of model magnetic field, the $\Delta B_{\perp 1}$ component is perpendicular to the model magnetic field and directed radially outward, and the $\Delta B_{\perp 2}$ component is azimuthally eastward. The field components $\Delta B_{\|}, \Delta B_{\perp 1}$, and $\Delta B_{\perp 2}$ are referred to as the compressional, radial, and azimuthal components, respectively.

In the second step we calculated the power spectral density for each component by using Fast Fourier Transform (FFT) analysis. A unit time window for FFT analysis was 25 minutes (500 data points) and this window was shifted in 5 -minute interval in each analysis. We could investigate pulsations in Pc 3-5 bands (10-600 seconds) because of the time window of 25 minutes and the time resolution of 3 seconds. Scanning the power spectral density of the $\Delta B_{\|}$component, we located the peak of the power spectrum. If the power of the peak was more than ten times as large as that of the background levels, we decided that a compressional pulsa- 


\section{MLT-ILAT Orbit distribution}

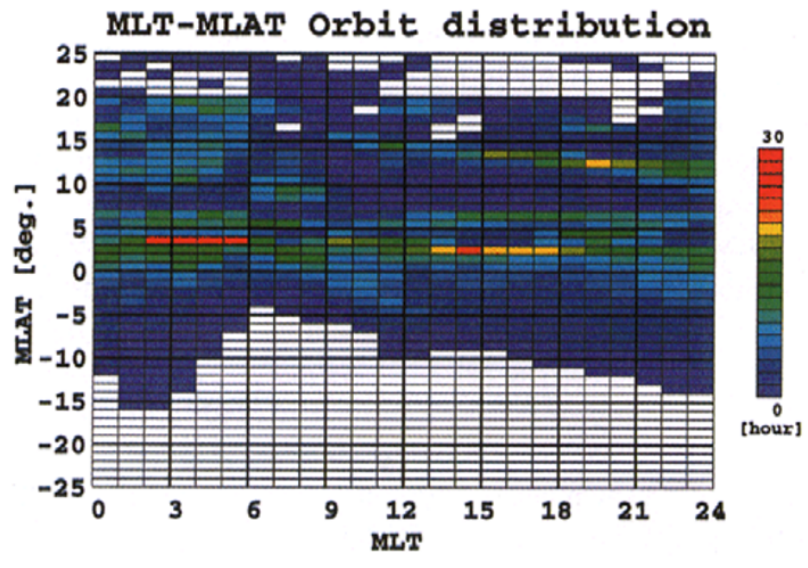

(a)

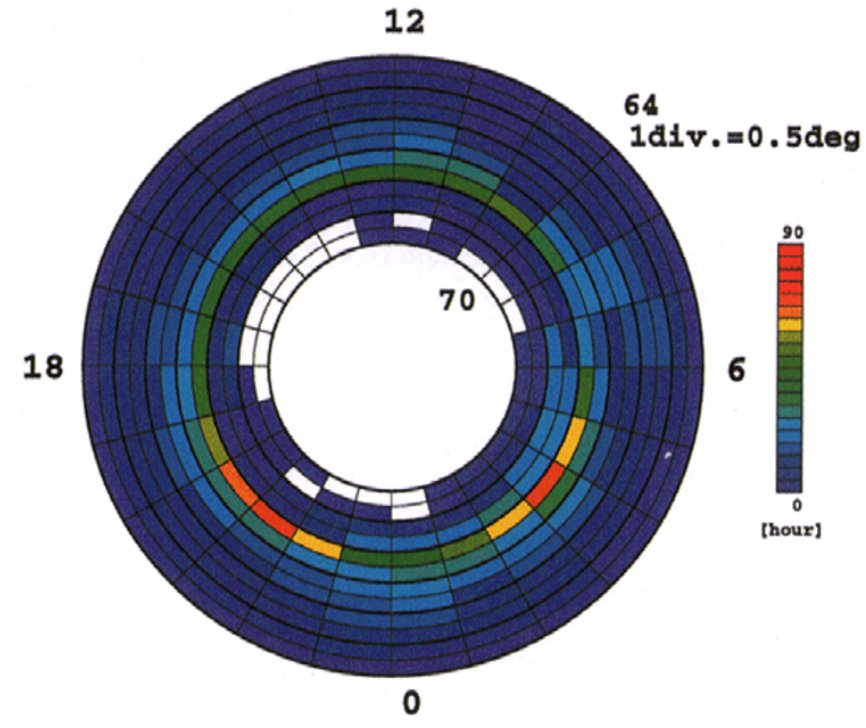

(b)

Plate 1. Distribution of data set in (a) MLT-MLAT plane and (b) MLT-ILAT plane. One mesh corresponds to (a) $1^{\circ}$ of MLAT by 1 hour of MLT, and (b) $0.5^{\circ}$ of ILAT by 1 hour of MLT. The color images show hours of observation time.

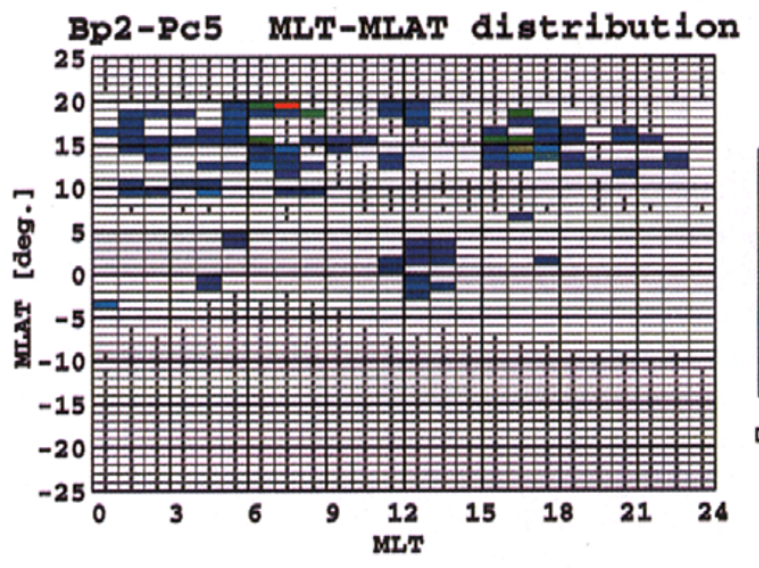

(a)

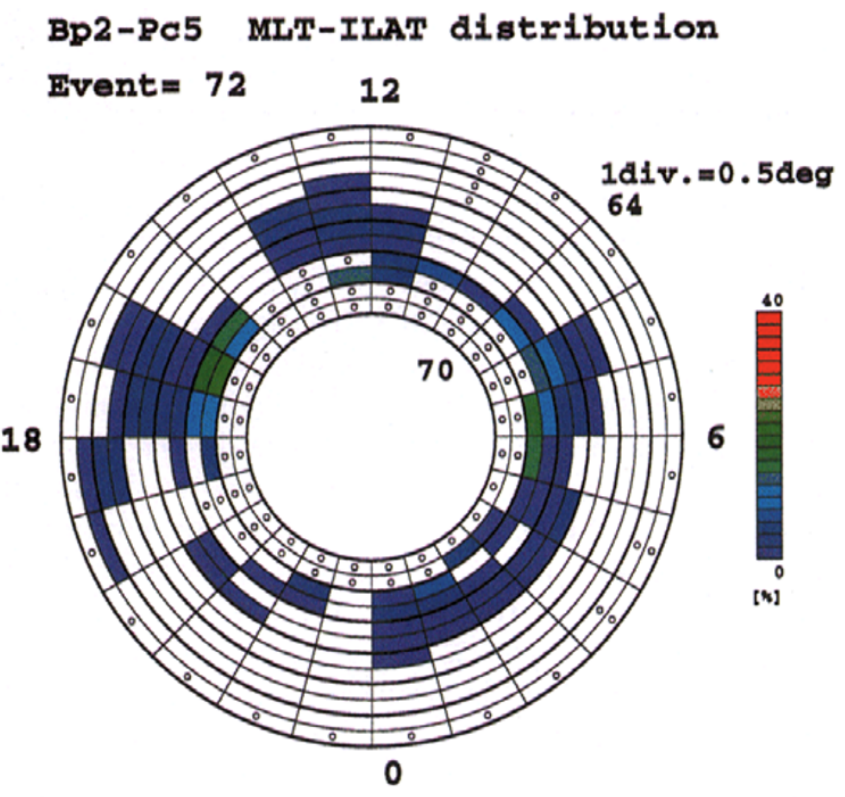

(b)

Plate 2. Distributions of occurrence probability of azimuthal Pc 5 pulsations in (a) MLT-MLAT plane with one mesh of $1^{\circ}$ of MLAT by 1 hour of MLT, and (b) MLT-ILAT plane with one mesh of $0.5^{\circ}$ of ILAT by 1 hour of MLT. The color images show occurrence probability of azimuthal Pc 5 pulsations which is defined as the appearance time of azimuthal Pc 5 divided by the observation time in each mesh. A small open circle in mesh represents that in that mesh no observation were made or observation time was small (less than 1.5 hours for (a), and less than 4.5 hours for (b)).

tion with the frequency at the spectral peak has appeared during the time interval of the window. To detect radial and azimuthal pulsations, the power spectrum densities of the $\Delta B_{\perp 1}$ and $\Delta B_{\perp 2}$ components were treated in the same way as the $\Delta B_{\|}$component. If pulsations were detected in successive time windows, we regarded them as one pulsation event.

In the last step we visually scanned the plot of the mag- 
netic field data and removed non-continuous events like Pi 2 pulsations from the pulsation lists. If a event had more than one component which satisfied the above criteria (i.e., the power of the spectral peak is more than ten times as large as that of the background), that event was treated to overlap more than one category according to the components. For example, a pulsation, in which the azimuthal and radial components satisfy the criteria, is counted as one event in both of the azimuthal and radial categories. The number of pulsations selected by the above procedure was shown in Table 1.

Table 1. The number of pulsations found in the data set. These pulsations were identified by the Fast Fourier Transform method and checked by visual scanning if they have continuous waveforms.

\begin{tabular}{lrcc}
\hline & \multicolumn{3}{c}{ Events } \\
\cline { 2 - 4 } & $\operatorname{Pc} 3$ & $\operatorname{Pc} 4$ & $\operatorname{Pc} 5$ \\
\hline$\Delta B_{\|}$(Compressional) & 59 & 148 & 18 \\
$\Delta B_{\perp 1}$ (Radial) & 119 & 221 & 37 \\
$\Delta B_{\perp 2}$ (Azimuthal) & 196 & 290 & 72 \\
\hline
\end{tabular}

\section{Statistical Results}

Based on the pulsation lists, we investigated occurrence distributions of pulsations classified by frequency ranges (Pc 3, Pc 4, and Pc 5) and components (compressional, radial, and azimuthal). Plate 2 is an example of the distributions of occurrence probability, which is for azimuthal Pc 5 pulsation. MLT-MLAT distribution of occurrence probability is shown in Plate 2(a), in which one mesh represents a region of $1^{\circ}$ of MLAT by 1 hour of MLT. Plate 2(b) gives MLTILAT distribution of occurrence probability with one mesh of $0.5^{\circ}$ of ILAT by 1 hour of MLT. The occurrence probability is defined as the appearance time of the pulsation divided by the observation time (shown in Fig. 1) in each mesh. A small open circle in mesh represents that in that mesh no observations were made or observation time was less than 1.5 hours in the MLT-MLAT plane and less than 4.5 hours in the MLAT-ILAT plane. The occurrence distributions for 9 kinds of pulsations shown in Table 1 were presented in the same format as Plate 2, and we have found prominent characteristics for the following pulsations: (1) azimuthal Pc 5 pulsation; (2) azimuthal Pc 3 pulsation; (3) radial Pc 4 pulsation on the dayside; (4) azimuthal Pc 4 pulsation on the nightside.

\subsection{Azimuthal Pc 5 pulsation}

Figure 1 gives an example of azimuthal Pc 5 pulsation observed by the ETS-VI satellite on 28 November 1995. The upper three panels are variations of the magnetic field in LMG coordinate system, and the bottom two panels show the location of the satellite in solar magnetic coordinate system. We notice a pulsation with period of about 3.2 minutes appeared from $0630 \mathrm{UT}$ to $0730 \mathrm{UT}$ in the $\Delta B_{\perp 2}$ component, that is, in the azimuthal component. This azimuthal Pc 5 pulsation appeared between 06 and 07MLT. As shown in the bottom right panel, the ETS-VI satellite was off the geomagnetic equator.
The distributions of occurrence probability of azimuthal Pc 5 pulsations are shown in Plate 2. The MLT-ILAT distribution (Plate 2(a)) shows that azimuthal Pc 5 pulsations mostly appear in the off equatorial region (around $15^{\circ} \mathrm{MLAT}$ ) except for an appearance near the geomagnetic equator at local noon. We could not conclude whether the azimuthal Pc 5 pulsations appear off the equator in the southern hemisphere, because ETS-VI did not pass the high latitude area of MLAT in the southern hemisphere. It is noticed that the Pc 5 pulsations occur frequently around $68^{\circ}-69^{\circ}$ ILAT of both $05-09$ MLT and 15-18MLT (Plate 2(b)), however the orbit of ETS-VI did not allow us to investigate whether the frequent occurrence regions of the azimuthal Pc 5 pulsations spread over higher latitude of ILAT. In the MLT-MLAT and MLT-ILAT regions where the azimuthal Pc 5 pulsations observed frequently, the occurrence probability of compressional and radial Pc 5 pulsations (not shown here) were much smaller than that of the azimuthal Pc 5 pulsations. This fact is consistent with the observation shown in Fig. 1.

Figure 2 shows $K p$ dependence of Pc 5 occurrence probability which was defined as the ratio of the number of the azimuthal Pc 5 pulsations in the MLT range of 04-10MLT and 15-20MLT to the number of data sets in each class of $K p$ index. It can be seen that generally the larger the value of $K p$ index, the more frequent the Pc 5 occurrence.

\subsection{Azimuthal Pc 3 pulsation}

An example of azimuthal Pc 3 pulsation, which was observed on 18 July 1995, is shown in Fig. 3. We can see fluctuations of the magnetic field with period of 20-30 seconds and with dominant component in $\Delta B_{\perp 2}$, that is, azimuthal Pc 3 pulsation. The azimuthal Pc 3 pulsation lasted for about 5 hours from 0600UT to 1100UT (magnetic field data after 0800UT are not shown here). ETS-VI was located near the geomagnetic equator at local noon.

The dynamic spectrum of the magnetic field data of Fig. 3 is presented in Plate 3. Each panel corresponds to the $\Delta B_{\|}$, $\Delta B_{\perp 1}$, and $\Delta B_{\perp 2}$ components. In $\Delta B_{\perp 2}$ there are harmonic waves which have mainly 2 frequency bands, high and low frequency. The high frequency wave has a frequency of about $45 \mathrm{mHz}$ around $0620 \mathrm{UT}$, and changes its frequency gradually to about $37 \mathrm{mHz}$ around $0740 \mathrm{UT}$. The low frequency wave has also changed its frequency from $20 \mathrm{mHz}$ to $17 \mathrm{mHz}$. The wave power of the high frequency wave is greater than that of the low frequency wave. We can notice other waves with a frequency of $65 \mathrm{mHz}$ from 0620UT to 0645UT and with a frequency of $29 \mathrm{mHz}$ from $0730 \mathrm{UT}$ to $0800 \mathrm{UT}$. As it was also seen in Fig. 3, wave power in the $\Delta B_{\perp 2}$ component are greater than those in the other two components.

The distributions of occurrence probability of the azimuthal Pc 3 pulsations are presented in Plate 4. It was found that the azimuthal Pc 3 pulsations were observed in the wide range of MLAT (Plate 4(a)). From Plate 4(b) we notice that Pc 3 appeared frequently in large MLT-ILAT region of 07$16 \mathrm{MLT}$ and $65^{\circ}-68.5^{\circ} \mathrm{ILAT}$, though the occurrence region of the azimuthal Pc 3 pulsations may spread over higher or lower latitude of ILAT. There is an occurrence peak around 09$13 \mathrm{MLT}$ and the occurrence probability becomes very small on the nightside (16-05MLT). 

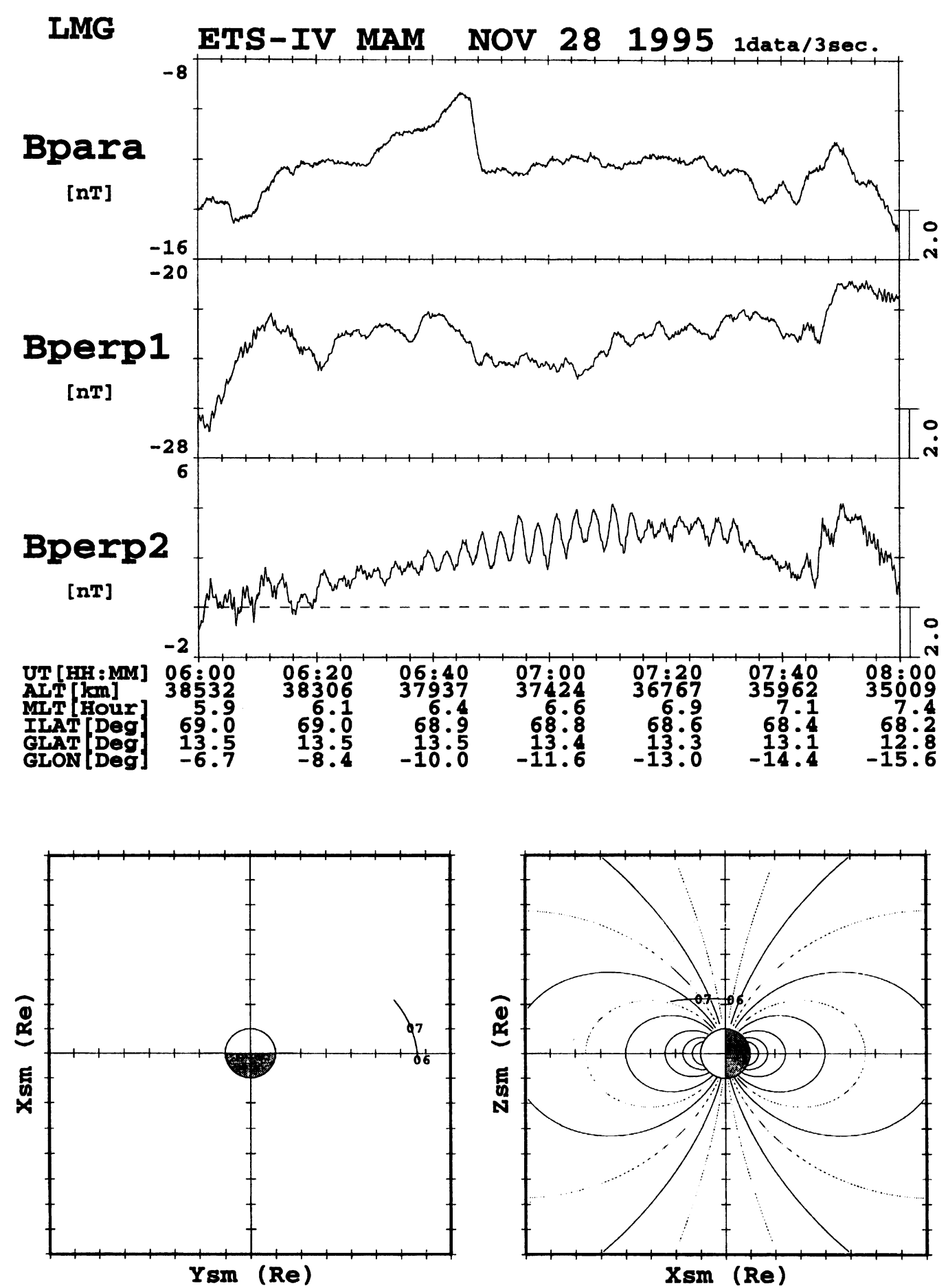

Fig. 1. An example of azimuthal Pc 5 pulsation observed by ETS-VI on 28 November 1995. The upper three panels are magnetic variations in LMG coordinate system, where the $\Delta B_{\|}$component is the tangential direction of model magnetic field, the $\Delta B_{\perp 1}$ component is perpendicular to the model magnetic field and directed radially outward, and the $\Delta B_{\perp 2}$ component is azimuthally eastward. The bottom two panels show the location of the satellite in solar magnetic coordinate system.

\subsection{Radial Pc 4 pulsation on the dayside}

Figure 4 shows an example of radial Pc 4 pulsation observed by ETS-VI on 26 July 1995 . Waves having two packet structures and periods of about 100 seconds can be easily seen in the $\Delta B_{\perp 1}$ (radial) component. Periods of the waves were almost constant. Amplitudes of the radial component were 3 times as large as those of the azimuthal component. It is noticed that the pulsation appeared near the geomagnetic equator on the dayside (14-15MLT).

The distributions of occurrence probability of the radial Pc 4 pulsations are given in Plates 5(a) and 5(b), which show the radial Pc 4 pulsations are frequently observed on the afternoon side (11-20MLT). Plate 5(a) shows that the radial Pc 4 pulsations appeared in the wide range of MLAT. It 


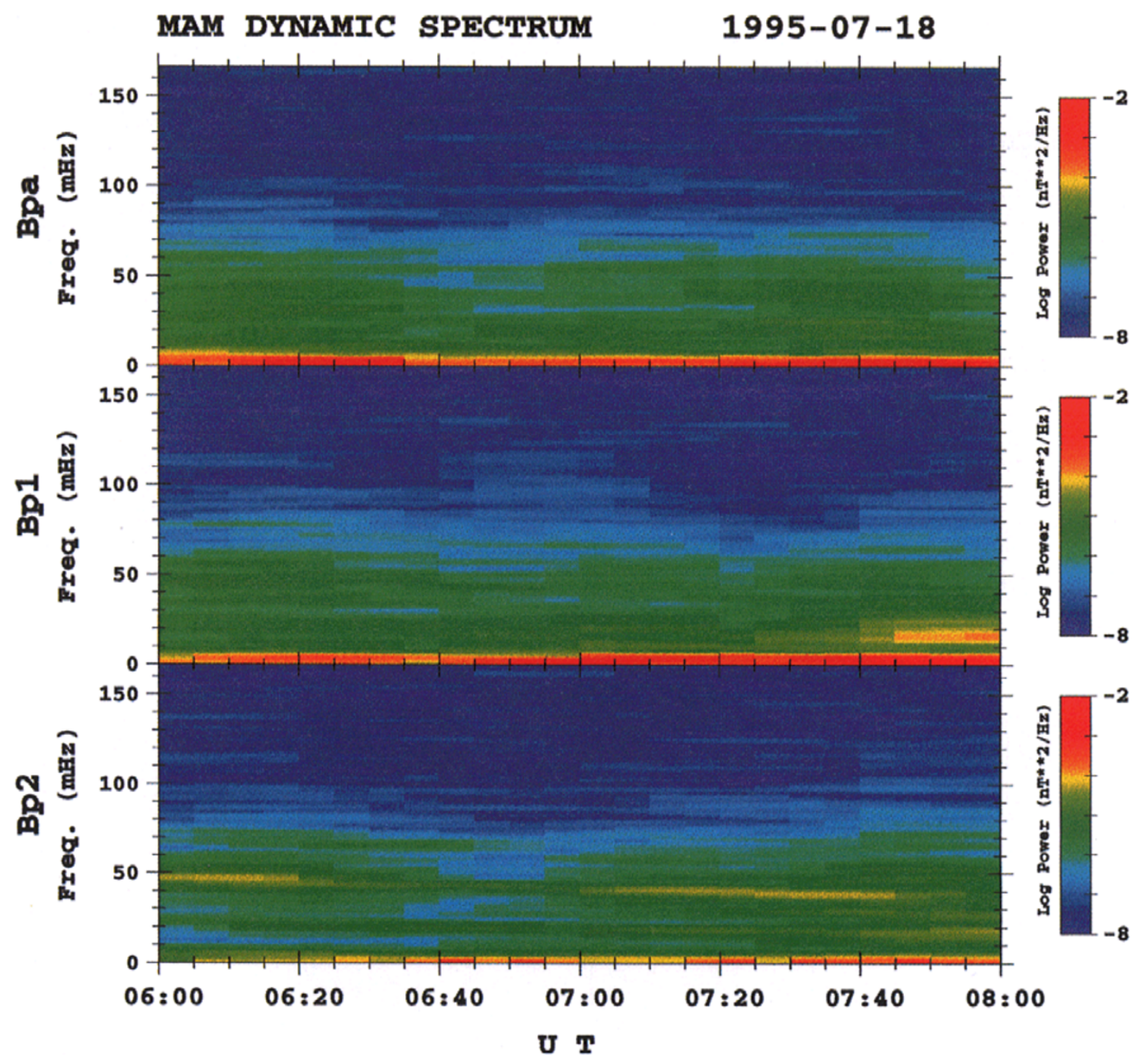

Plate 3. Dynamic spectrum of the magnetic field data obtained by ETS-VI from 06 to 08UT on 18 July 1995.

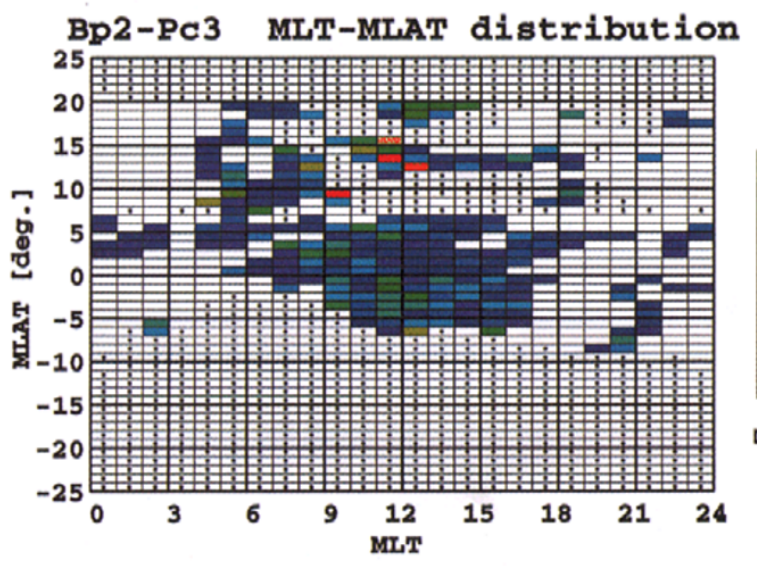

(a)
Bp2-PC3 MLT-ILAT distribution

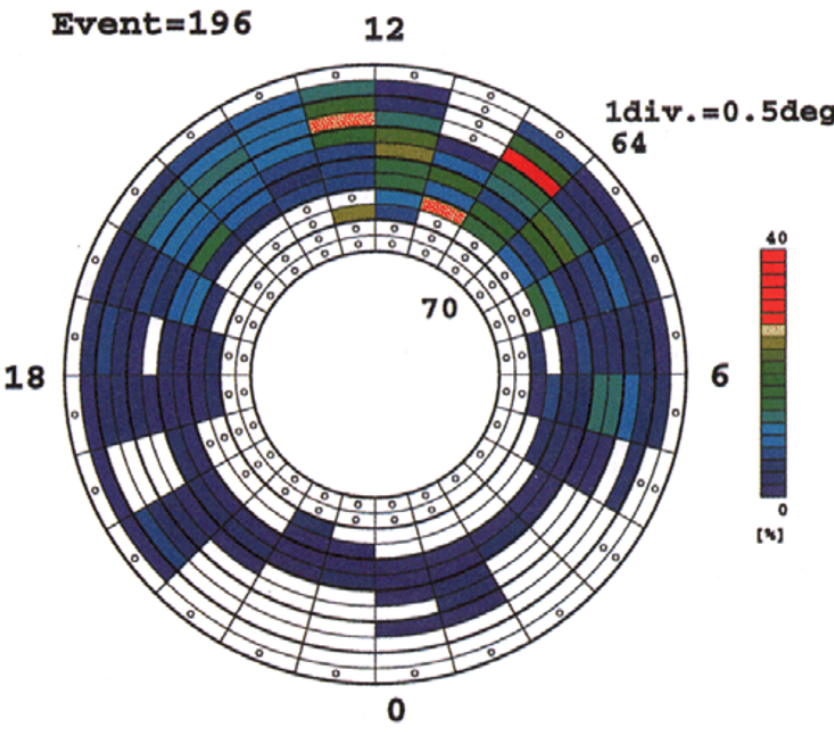


Bp1-PC4 MLT-ILAT distribution

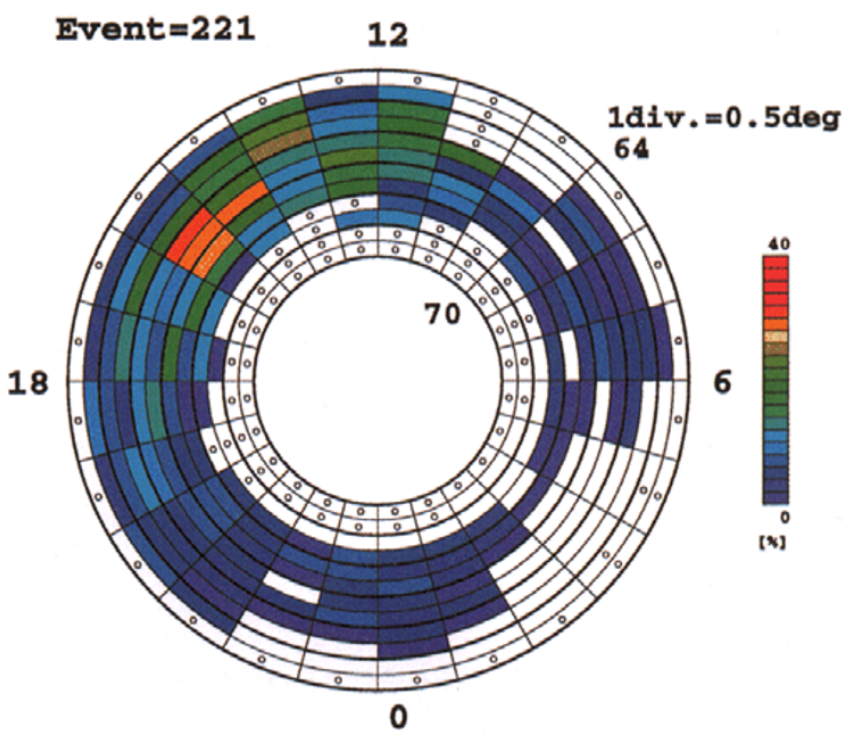

(b)

Plate 5. Same as in Plate 2 except for radial Pc 4 pulsations on the dayside.

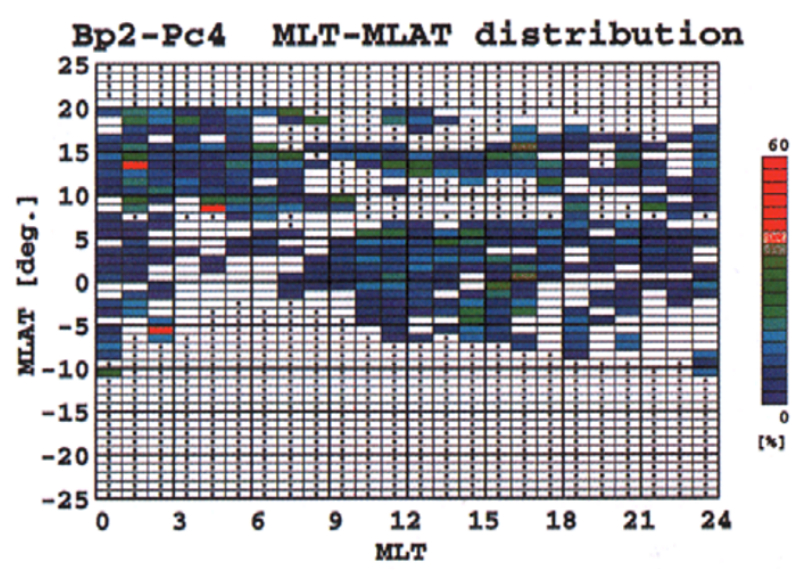

(a)

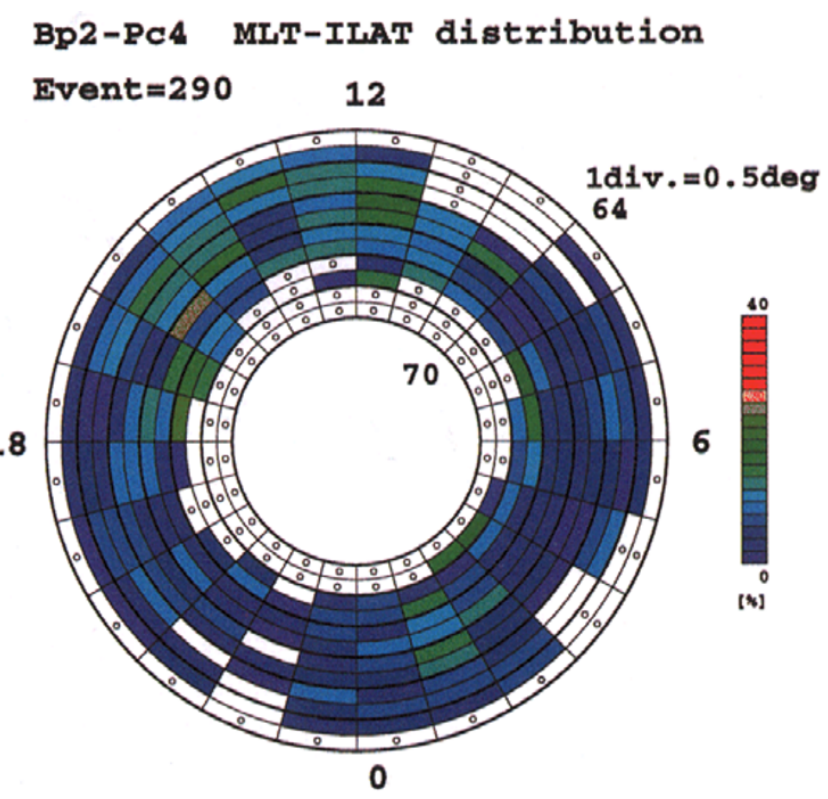

(b)

Plate 6. Same as in Plate 2 except for azimuthal Pc 4 pulsations on the nightside.

was found in Plate 5(b) that the occurrence peak lies in the MLT-ILAT area of $14-16 \mathrm{MLT}$ and $66^{\circ}-67.5^{\circ} \mathrm{ILAT}$. On the night and morning side (20-09MLT), the occurrence probability was very small. Comparing Plate 5 with Plate 6 which shows the distributions of occurrence probability of the azimuthal Pc 4 pulsation, we notice that Pc 4 pulsations on the afternoon side (11-20MLT) appeared more frequently in the radial component than in the azimuthal component.
This result is consistent with the larger amplitude of the Pc 4 pulsation in the radial component shown in Fig. 4.

\subsection{Azimuthal Pc 4 pulsation on the nightside}

As described previously, Pc 4 pulsations on the dayside (11-20MLT) are polarized radially. However, on the nightside, we found from Plate 6 that the azimuthal Pc 4 pulsations have frequent occurrence in the MLT range of 23-04MLT, where the radial $\operatorname{Pc} 4$ pulsations have a small occurrence 


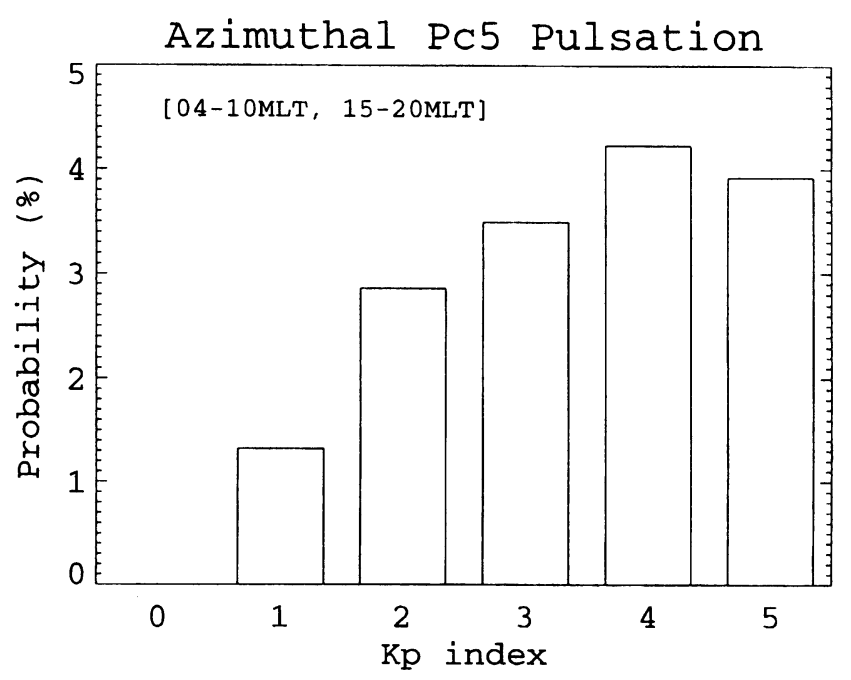

Fig. 2. $K p$ dependence of Pc 5 occurrence which was defined as the ratio of the number of the azimuthal Pc 5 pulsations in the MLT range of 04-10MLT and 15-20MLT to the number of data set in each class of $K p$ index.

probability. There is an occurrence peak of the azimuthal Pc 4 pulsations in the MLT-ILAT area of 01-02MLT and $66^{\circ}-68.5^{\circ}$ ILAT (Plate 6(b)). Plate 6(a) shows that occurrence probability on the nightside is larger in the high latitude of MLAT than in the low latitude of MLAT.

An example of azimuthal Pc 4 pulsation on the nightside, which was observed on 29 January 1996, is shown in Fig. 5. Waves with periods of 80-100 seconds and larger amplitude in the azimuthal component started to oscillate from about 1302UT, 1312UT, and 1332UT, which are indicated by arrows. Each of these waves lasted for about 10 minutes. The observation was made around 02-03MLT in the off equatorial region, which supports the result of Plate 6(a). In the first and second events, we found short-lived disturbances with small amplitudes in the compressional component, which are indicated by horizontal lines. However, there was no clear disturbances in the compressional component at 1332UT when the third event started.

Judging from the distribution of occurrence probability and waveform, we consider that the azimuthal Pc 4 pulsations on the nightside are the same phenomena as TTW reported by Takahashi et al. (1996) and QPO reported by Saka et al. (1996a).

\section{Discussion}

\subsection{Azimuthal Pc 5 pulsation}

Plate 2(b) indicates that the frequent occurrence regions of the azimuthal Pc 5 pulsations are around both 05-09MLT and $15-18 \mathrm{MLT}$ for $68^{\circ}-69^{\circ}$ ILAT. Takahashi and McPherron (1984), Kokubun (1985), and Kokubun et al. (1989) found that azimuthally polarized Pc 5 pulsations have a large peak of occurrence probability on the dawn side, using geosynchronous satellite $\left(\sim 67^{\circ}\right.$ ILAT) data. Anderson et al. (1990a) found that the occurrence distribution of fundamental toroidal waves in Pc 5 band observed by the AMPTE/CCE satellite has a strong bias towards the dawn flank for $L=8-9$.
Kokubun (1985) and Kokubun et al. (1989) found that there is a secondary small peak of occurrence probability in the afternoon (around 15LT). Their results are generally consistent with the distribution of occurrence probability obtained by this study. Nosé et al. (1995) and Potemra and Blomberg (1996) examined the Pc 5 waves in the high-latitude region of ILAT, taking advantage of polar orbiting satellites. Nosé et al. (1995) analyzed the magnetic field data from the DE-1 satellite and found that the transverse Pc 5 pulsations occur most frequently around $72^{\circ}$ ILAT between 08 and 10MLT. From morning to noon, Pc 5 pulsations observed by the Viking satellite are polarized primarily in the east-west direction (Potemra and Blomberg, 1996). The Pc 5 pulsations occurred $2^{\circ}-5^{\circ}$ equatorward of the interface of the region $1 /$ region 2 Birkeland currents (around $70^{\circ}-73^{\circ} \mathrm{MLAT}$ ) (see Potemra and Blomberg, 1996, Fig. 8). The average wave amplitudes were larger near 09-10MLT (about $40 \mathrm{nT}$ ) than those during the afternoon (about $25 \mathrm{nT}$ ). This indicates that Pc 5 pulsations are identified more readily in the prenoon region. The distribution of occurrence probability of the azimuthal Pc 5 pulsations obtained by the ETS-VI and geosynchronous satellites, which showed two occurrence peaks on the dawn and dusk sides, are consistent with the occurrence distribution by Nosé et al. (1995) and Potemra and Blomberg (1996) in the low latitude region. Thus we consider that the frequent occurrence regions in Plate 2(b) spread to the MLTILAT region higher than $70^{\circ}$ ILAT around 08-10MLT.

Azimuthal Pc 5 pulsations have been considered as the fundamental mode of the standing Alfvén waves (e.g., Hughes, 1994). This idea is supported by the statistical results shown in Plate 2(a), which indicate the frequent occurrence of the azimuthal Pc 5 pulsations off the geomagnetic equator, because the fundamental mode of the standing Alfvén wave has a node in the geomagnetic equator. Assuming the wave equation (Cummings et al., 1969) and the plasma mass density on the dayside (Takahashi and Anderson, 1992), we obtained the eigenperiod of 170-200 seconds for the fundamental mode at $68^{\circ}-69^{\circ} \mathrm{ILAT}$. The calculated period is almost the same as the period of observed wave (about 3.2 minutes) shown in Fig. 1.

It was found that the azimuthal Pc 5 pulsations generally occur more frequently when the value of $K p$ index becomes larger (Fig. 2). $K p$ index was thought to be correlated with the solar wind velocity (Snyder et al., 1963; Olbert, 1968). Thus the frequent occurrence of the azimuthal Pc 5 pulsations might be associated with the high-speed solar wind. This result is consistent with those of Kokubun et al. (1989) and Nosé et al. (1995). Anderson et al. (1990b) found that toroidal fundamental pulsations are enhanced for high solar wind velocity. Thus we suggest the Kelvin-Helmholtz instability on the magnetopause for the energy source of Pc 5 pulsations.

\subsection{Azimuthal Pc 3 pulsation}

The azimuthal Pc 3 pulsations have frequent occurrence distribution from 07 to 16 MLT for $65^{\circ}-68.5^{\circ} \mathrm{ILAT}$, and a strong occurrence peak in the prenoon sector (09-13MLT) as shown in Plate 4(b). Arthur and McPherron (1977) found a strong midmorning peak in the occurrence distribution of Pc 3 pulsations observed by the ATS-1 geosynchronous satellite. Analyzing data from the geosynchronous satellites, 

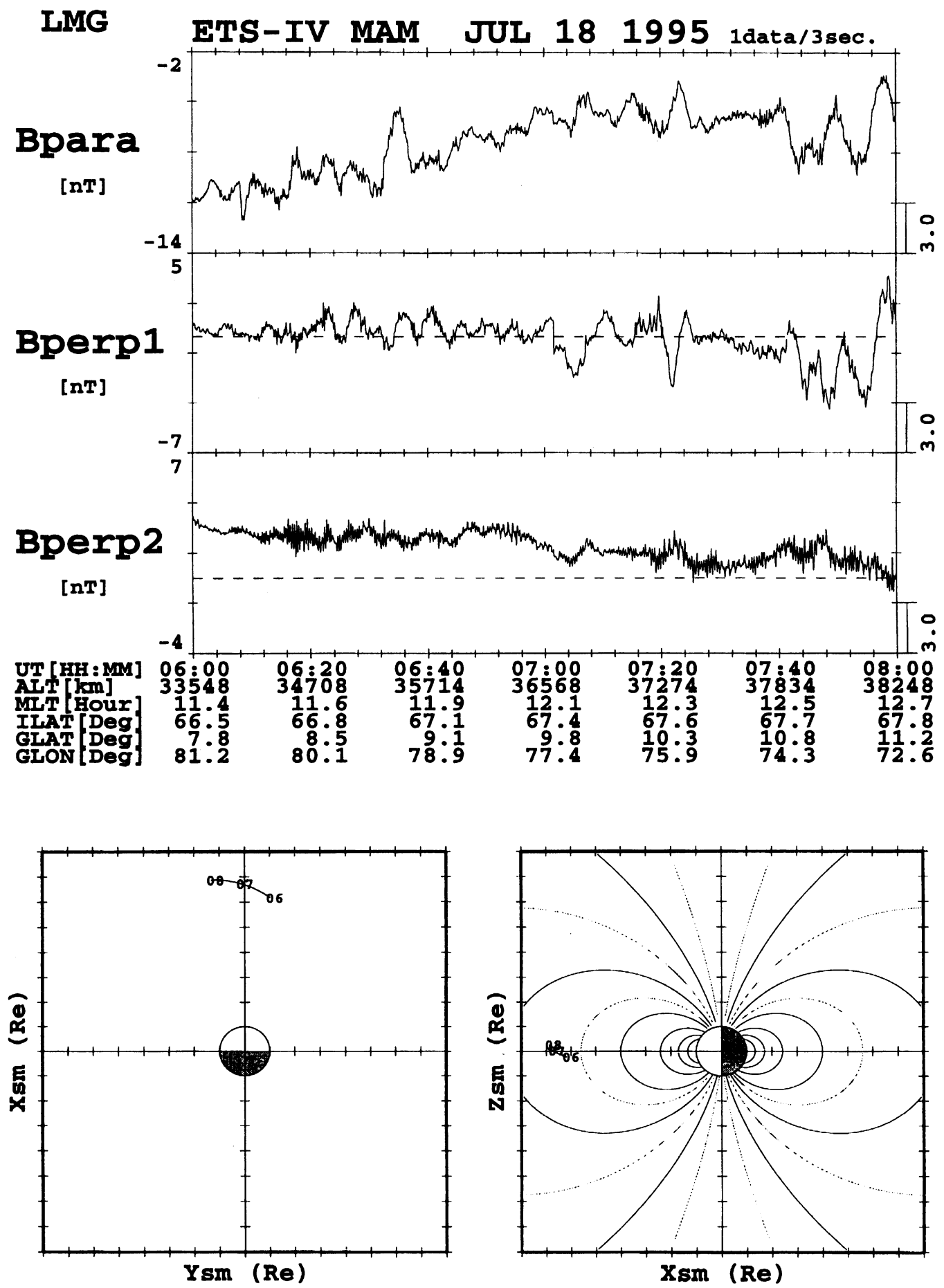

Fig. 3. An example of azimuthal Pc 3 pulsation observed on 18 July 1995. Format is the same as for Fig. 1.

Takahashi and McPherron (1984) presented occurrence probability of the azimuthally polarized harmonic waves, which shows a high occurrence on the dayside and a peak before local noon. Anderson et al. (1990a) showed that harmonic toroidal waves in Pc 3 bands observed by AMPTE/CCE are dominant on the dayside and occur frequently in the morning. Our result is consistent with the occurrence distributions obtained by the previous studies. Anderson et al. (1990a) exhibited besides that intense Pc 3 pulsations have an uniform distribution in local time from 06 to $16 \mathrm{MLT}$, suggesting that the morning side bias of occurrence distribution in the overall Pc 3 events may result from masking by other pulsations in the afternoon.

The continuous frequency change of the azimuthal Pc 3 pulsation seen on 18 July 1995 (Plate 3) is thought to be caused by the change of the satellite location, as reported by Engebretson et al. (1986). We notice from Fig. 3 that the ETS-VI satellite was moving away from the earth. The observed frequency of the harmonic waves is expected to decrease, because the length of the local geomagnetic field line 

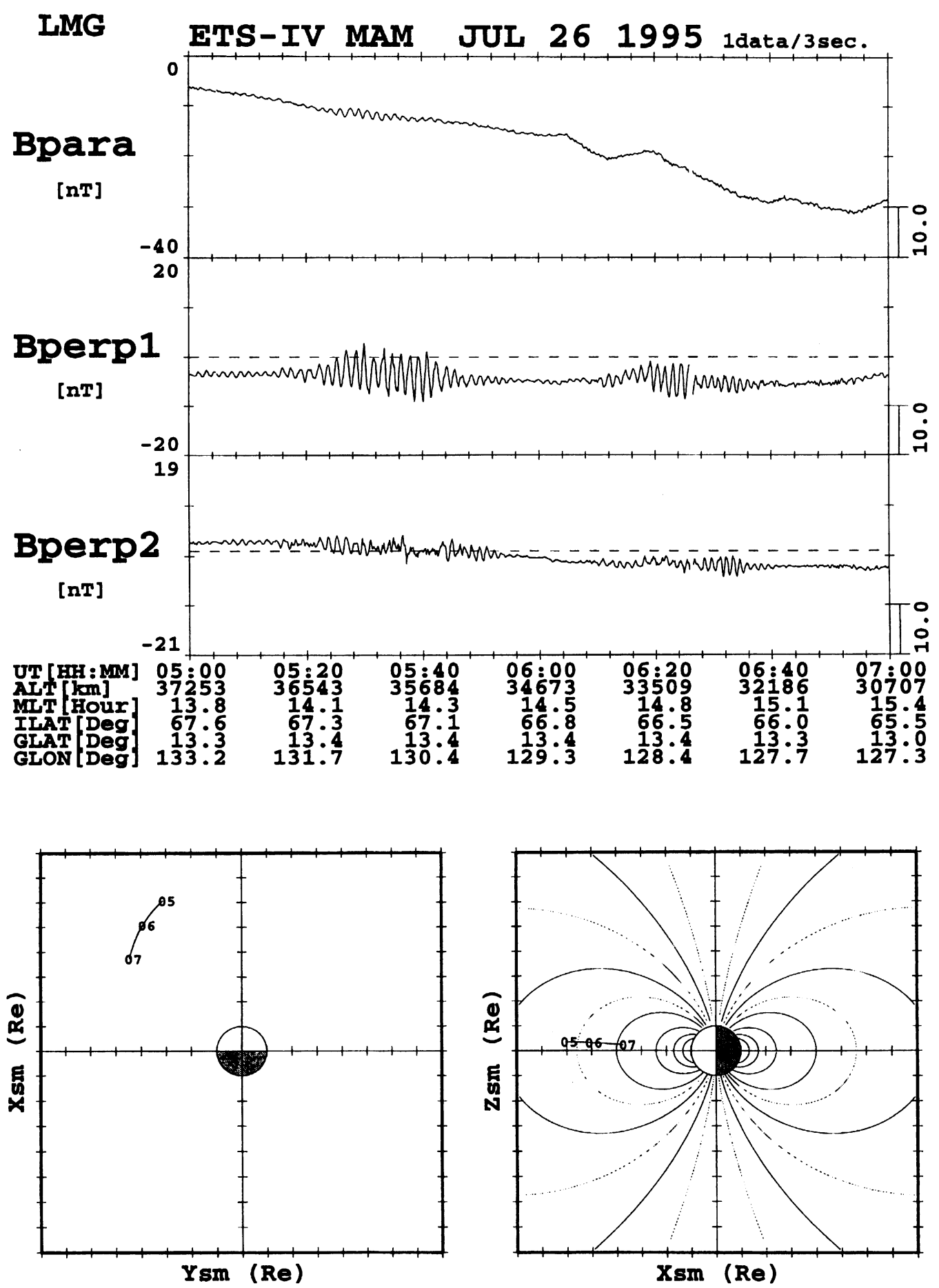

Fig. 4. An example of radial Pc 4 pulsation on the dayside observed on 26 July 1995. Format is the same as for Fig. 1.

becomes long. We calculated the frequencies of the harmonic waves for ILAT $=66.8^{\circ}$ and $67.7^{\circ}$, which correspond to the values of ILAT at 0620UT and 0740UT, respectively. The wave equation by Cummings et al. (1969) with the plasma mass density model by Takahashi and Anderson (1992) was solved numerically. The results are shown in Table 2 . The high frequency wave, which changed its frequency from 45 $\mathrm{mHz}$ to $37 \mathrm{mHz}$, is concluded as the fifth harmonic wave. The low frequency wave with continuous frequency change from $20 \mathrm{mHz}$ to $17 \mathrm{mHz}$ might be the second harmonic waves.
The other two waves, which appeared with a frequency of $65 \mathrm{mHz}$ around 0630UT and with a frequency of $29 \mathrm{mHz}$ around $0740 \mathrm{UT}$, are thought to be the 7 th and 4 th harmonic waves, respectively. The two main frequency bands involved in the azimuthal Pc 3 pulsation, that is, the high $(\sim 40 \mathrm{mHz})$ and low $(\sim 20 \mathrm{mHz})$ frequency bands, are also found in the results by Arthur and McPherron (1977).

\subsection{Radial Pc 4 pulsation on the dayside}

The radially polarized Pc 4 pulsations have been investigated by many authors using satellites (Arthur and 

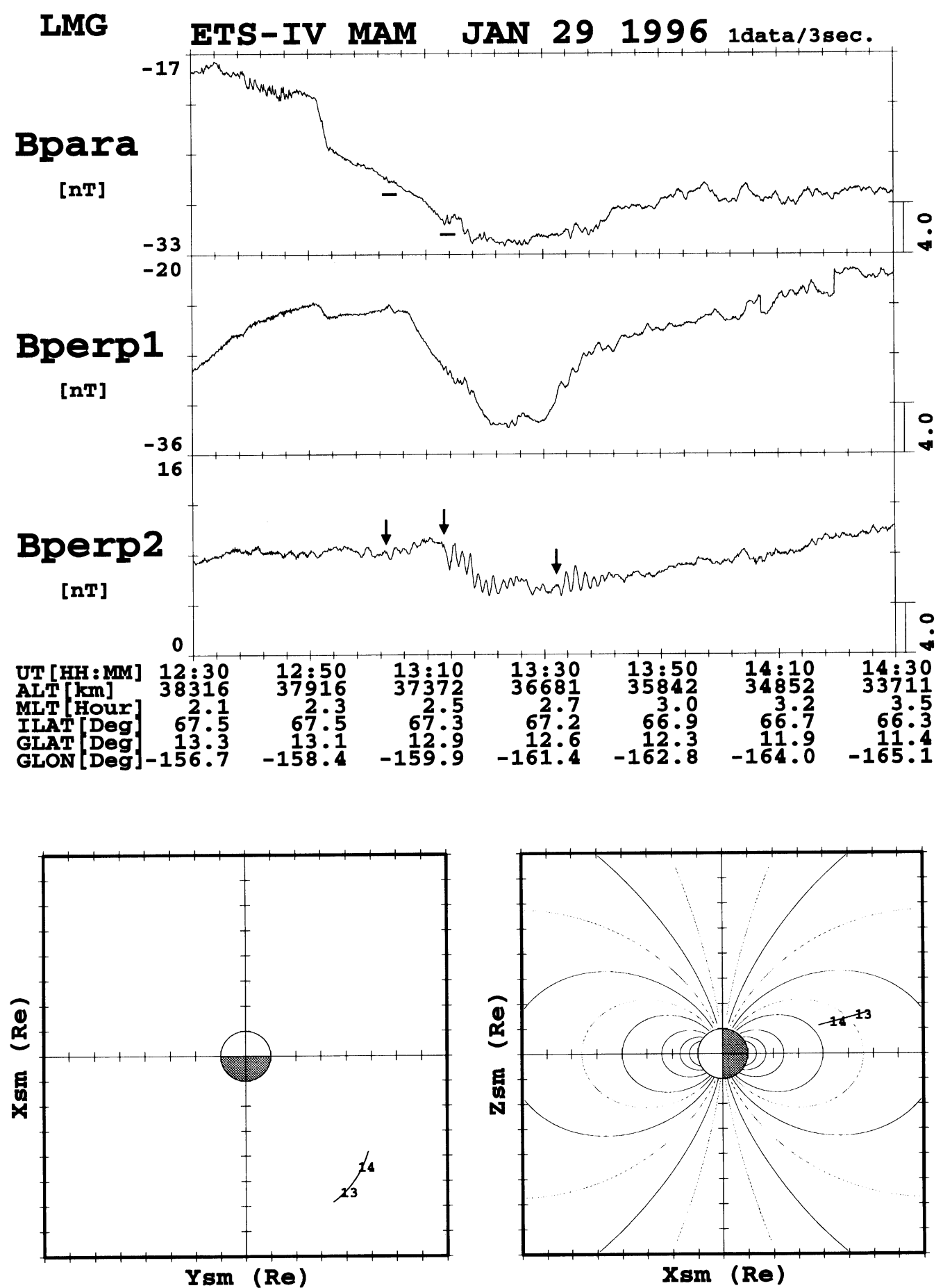

Fig. 5. An example of azimuthal Pc 4 pulsation on the nightside observed on 29 January 1996. Azimuthal Pc 4 pulsations started to oscillate from about 1302UT, 1312UT, and 1332UT, which are indicated by arrows. Format is the same as for Fig. 1.

McPherron, 1981; Takahashi and McPherron, 1984; Anderson et al., 1990a; Takahashi and Anderson, 1992). The radial Pc 4 pulsations are found to be the phenomena from the afternoon to dusk sector, primarily in the early afternoon. The occurrence distribution exhibited a shift from $L<7$ on the dayside to $L>7$ at midnight (Anderson et al., 1990a). Our results, which showed the frequent occurrence from 11 to $20 \mathrm{MLT}$, are in agreement with the results of the previous studies.
The radial Pc 4 pulsations on the afternoon side were thought to be generated by bounce resonance (Arthur and McPherron, 1981; Takahashi and McPherron, 1984; Anderson et al., 1990a; Takahashi and Anderson, 1992). Takahashi and McPherron (1984) and Anderson et al. (1990a) suggested that the spatial gradient in ion density is responsible for driving the waves through bounce resonance. We can not conclude the excitation mechanisms for the radial Pc 4 pulsations, because the particle data were not available 
Table 2. The frequencies of the harmonic waves for $66.8^{\circ}$ ILAT and $67.7^{\circ}$ ILAT. The wave equation by Cummings et al. (1969) was solved numerically with the plasma mass density model by Takahashi and Anderson (1992).

\begin{tabular}{ccc}
\hline & \multicolumn{2}{c}{ Eigenfrequency $(\mathrm{mHz})$} \\
\cline { 2 - 3 } Harmonic & $66.8^{\circ} \mathrm{ILAT}$ & $67.7^{\circ} \mathrm{ILAT}$ \\
\hline 1 & 7.65 & 6.39 \\
2 & 17.4 & 14.5 \\
3 & 27.2 & 22.7 \\
4 & 36.8 & 30.9 \\
5 & 46.3 & 39.1 \\
6 & 55.6 & 47.2 \\
7 & 65.8 & 55.6 \\
\hline
\end{tabular}

in this study.

\subsection{Azimuthal Pc 4 pulsation on the nightside}

We have found the appearance of the azimuthally polarized Pc 4 pulsations on the nightside (23-04MLT). There were few works which dealt with this kind of pulsation, except for Takahashi et al. (1996) and Saka et al. (1996a, b). We will discuss the following four subjects related to the azimuthal Pc 4 pulsations on the nightside (the nightside Pc 4 pulsations). (1) Are the nightside Pc 4 pulsations different phenomena from Pi 2 pulsations in the magnetosphere? (2) Are they standing Alfvén waves? (3) Is it possible for the nightside Pc 4 pulsations to continue oscillating for long time ( $\sim 10$ minutes)? (4) What is/are their energy source? In what mechanism(s) are they excited? Finally, the nightside Pc 4 pulsations will be discussed in relation to results of the previous studies.

4.41 Nightside Pc 4 pulsations and Pi 2 pulsations in the magnetosphere In previous studies Pi 2 pulsations in the magnetosphere are considered to be dominant in the compressional and radial components (Lin and Cahill, 1975; Sakurai and McPherron, 1983; Takahashi et al., 1995). Pi 2 pulsations observed by Explorer 45 near $L=5$ had a strong compressional component (Lin and Cahill, 1975). Sakurai and McPherron (1983) studied Pi 2 pulsations observed by the geosynchronous satellite and found that they had a significant compressional component in most cases. Within the magnetic latitude $\pm 16^{\circ}$ of the AMPTE/CCE satellite the Pi 2 pulsations are dominated by the radial and compressional components (Takahashi et al., 1995). On the other hand, The Pc 4 pulsations on the nightside have continuous waveforms and are dominant in the azimuthal component as can be seen in Fig. 5. Thus we suggest that the nightside Pc 4 pulsations differ from the Pi 2 pulsations in the magnetosphere.

The local time dependence of Pi 2 occurrence in the magnetosphere was investigated by Sakurai and McPherron (1983) and Singer et al. (1983). The most probable occurrence time of Pi 2 pulsations observed by the ATS- 6 satellite is $21 \mathrm{LT}$, with more events seen premidnight than postmidnight (Sakurai and McPherron, 1983). The distributions of Pi 2 occurrence at GOES-2 and -3 orbits have peaks around

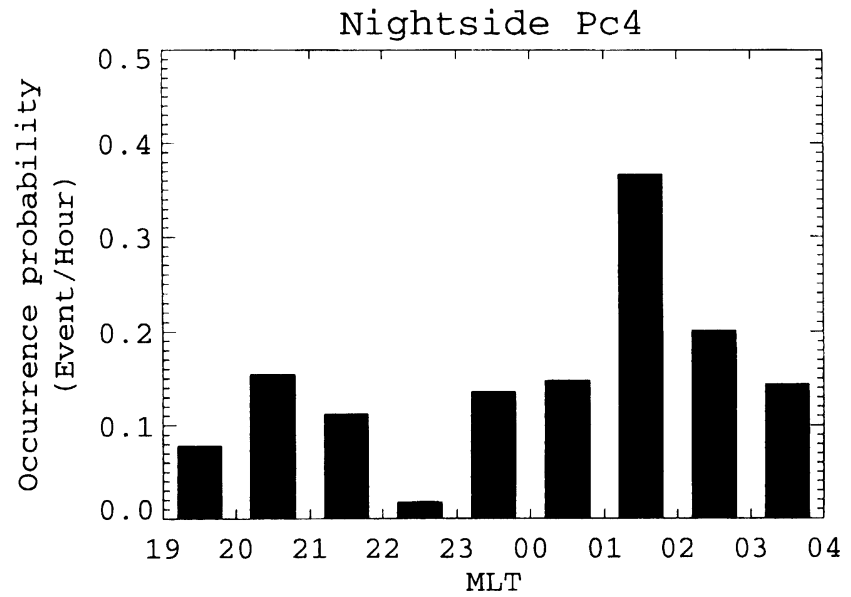

Fig. 6. Dependence of the nightside Pc 4 pulsation occurrence on MLT. Occurrence probability was defined as the number of pulsation divided by the observation time in each bin of MLT.

1-2 hours prior to local midnight (Singer et al., 1983). Both of their results showed that occurrence probability of Pi 2 pulsations decreases generally as the satellite moves to the dusk or the early morning side. We found 80 events of Pc 4 pulsations in the night sector (19 events for 19-23MLT, and 61 events for 23-04MLT), which have much power in the azimuthal component than in the radial and compressional components. The dependence of the nightside Pc 4 pulsation occurrence on MLT was investigated (Fig. 6). We defined occurrence probability as the number of pulsations divided by the observation time in each bin of MLT. As shown in Fig. 6, the nightside Pc 4 pulsations have frequent occurrence from 23 to 04MLT and an occurrence peak around 01-02MLT. Occurrence probability before midnight (19-23MLT) is generally smaller than that after midnight (23-04MLT). The nightside Pc 4 pulsations are seldom observed at the range of 22-23MLT, where Pi 2 pulsations have an occurrence peak. It is obvious that there are differences between the local time occurrence distribution of the nightside Pc 4 pulsations and that of the Pi 2 pulsations. The result of Fig. 6 also confirms that the nightside Pc 4 pulsations are different phenomena from the Pi 2 pulsations in the magnetosphere.

4.42 Standing Alfvén wave We investigated the ILAT dependence of the periods of the nightside Pc 4 pulsations for 23-04MLT (Fig. 7). If we ignore a data point with a parenthesis, there seems to be a tendency that the higher the value of ILAT, the longer the periods of the nightside Pc 4 pulsations. The broken lines show the eigenperiods of the standing Alfvén waves for azimuthal waves calculated from the wave equation of Cummings et al. (1969). The plasma density is assumed to be half the values of Takahashi and Anderson (1992) which gives the mass density model in the dayside magnetosphere (09-12MLT), because the observation of the Ogo-5 satellite shows that $\mathrm{H}^{+}$density in the prenoon sector is a few to ten times larger than that in the postmidnight sector (Chappell et al., 1970). The broken line which indicates the eigenperiod for the fundamental mode fits mostly the observation of the ETS-VI satellite. We consider that the fundamental mode of the standing Alfvén waves 


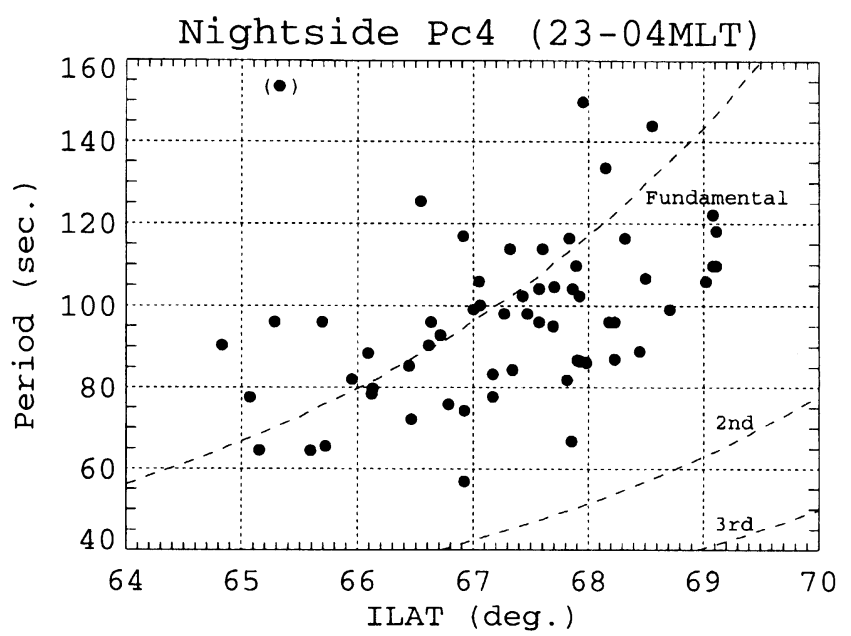

Fig. 7. ILAT dependence of the periods of the nightside Pc 4 pulsations for 23-04MLT. The broken lines show the eigenperiods of the standing Alfvén waves for azimuthal waves calculated from the wave equation of Cummings et al. (1969) with plasma density model which has half the values of Takahashi and Anderson (1992).

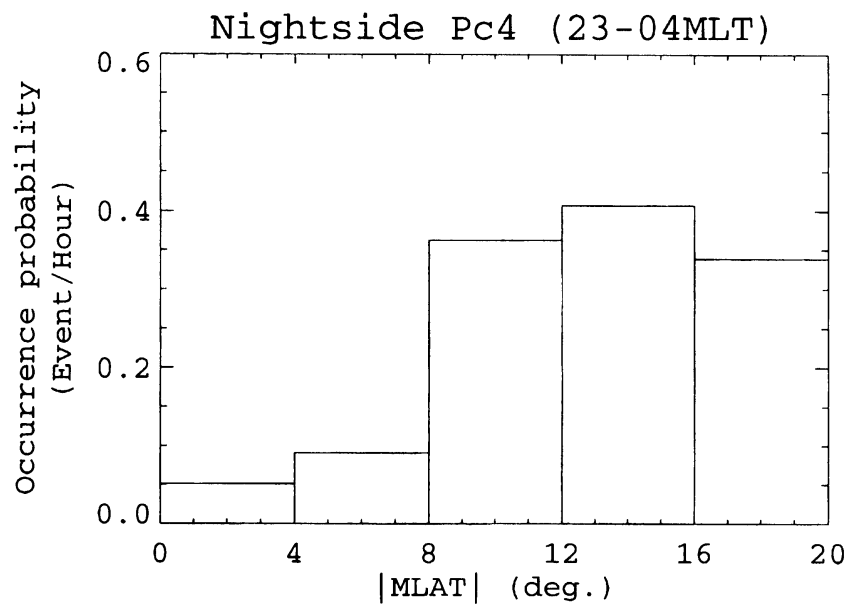

Fig. 8. Dependence of occurrence of the nightside Pc 4 pulsations on |MLAT|. Occurrence probability was defined as the number of nightside Pc 4 pulsation divided by the observation time in each bin of |MLAT|.

is most plausible for the nightside Pc 4 pulsations.

Figure 8 shows the dependence of occurrence of the nightside Pc 4 pulsations on the absolute value of MLAT. Occurrence probability was defined as the number of nightside Pc 4 pulsation divided by the observation time in each bin of $\mid$ MLAT $\mid$. In the low latitude of MLAT ( $\mid$ MLAT $\mid<8^{\circ}$ ) the nightside Pc 4 pulsations were less observed. Occurrence probability in the high latitude of MLAT (|MLAT| > $8^{\circ}$ ) is about 4 times as large as that in the low latitude of MLAT $\left(\mid\right.$ MLAT $\left.\mid<8^{\circ}\right)$. This fact, which can be also seen in Plate 6(a), supports that the nightside Pc 4 pulsations are the fundamental mode of the geomagnetic field line oscillation.

It should be noted that the nightside Pc 4 pulsations are expected to have periods longer than 150 seconds, which fall in the period range of Pc 5 pulsations, at larger radial distance than ETS-VI apogee, because they are considered to be the fundamental mode of standing Alfvén waves.

4.43 Oscillation lasting for long time ( $\sim 10$ minutes) The nightside $\mathrm{Pc} 4$ pulsations were observed in continuous waveforms lasting for about 10 minutes (Fig. 5). If the nightside Pc 4 pulsations are excited by impulsive energy source, which will be discussed later, duration of the pulsations depends on Joule dissipation in the ionosphere. Here we calculated the damping rate of the nightside Pc 4 pulsation which were derived by Ellis and Southwood (1983) with an emprical model of the ionospheric Pedersen conductivity (Spiro et al., 1982).

Figure 9 shows ILAT dependence of the height-integrated Pedersen conductivity by Spiro et al. (1982) in the postmidnight sector (01-02MLT) for $300 \mathrm{nT}<\mathrm{AE} \leq 600 \mathrm{nT}$ condition. The height-integrated Pedersen conductivity has large value ( $>8 \mathrm{mho}$ ) in the ILAT range of $63^{\circ}-67^{\circ}$ ILAT and a peak of 10.4 mho at $65^{\circ}$ ILAT. Ellis and Southwood (1983) gives the damping rate of standing Alfvén waves $\gamma$ by the form:

$$
\gamma=\frac{V_{A}}{l} \ln \left\{\left|\frac{\Sigma_{A}-\Sigma_{P}}{\Sigma_{A}+\Sigma_{P}}\right|^{-1}\right\},
$$

where $V_{A}$ is the Alfvern speed, $l$ is the length of the geomagnetic field line, $\Sigma_{A}$ is the Alfvén conductivity, and $\Sigma_{P}$ is the height-integrated Pedersen conductivity. If we take $V_{A} \sim 3000 \mathrm{~km} / \mathrm{s}, l \sim 1.2 \times 10^{8} \mathrm{~m}$, and $\Sigma_{A} \sim 0.5 \mathrm{~S}$, the duration of the pulsation $\tau\left(=\gamma^{-1}\right)$ are calculated as a function of $\Sigma_{P}$ (Table 3). Table 3 indicates that pulsations around $63^{\circ}-67^{\circ}$ ILAT on the nightside last for $6-7$ minutes. Because the duration of the pulsation $\tau$ is defined as the time in which the wave amplitude decays to $\frac{1}{e}$ (i.e., about $37 \%$ ) of its initial amplitude, we can expect that pulsations appear with observable amplitude for a little longer than the estimated duration (6-7 minutes). This result is rather consistent with the observation.

4.44 Energy source and excitation mechanism We expect magnetospheric substorms as a likely excitation

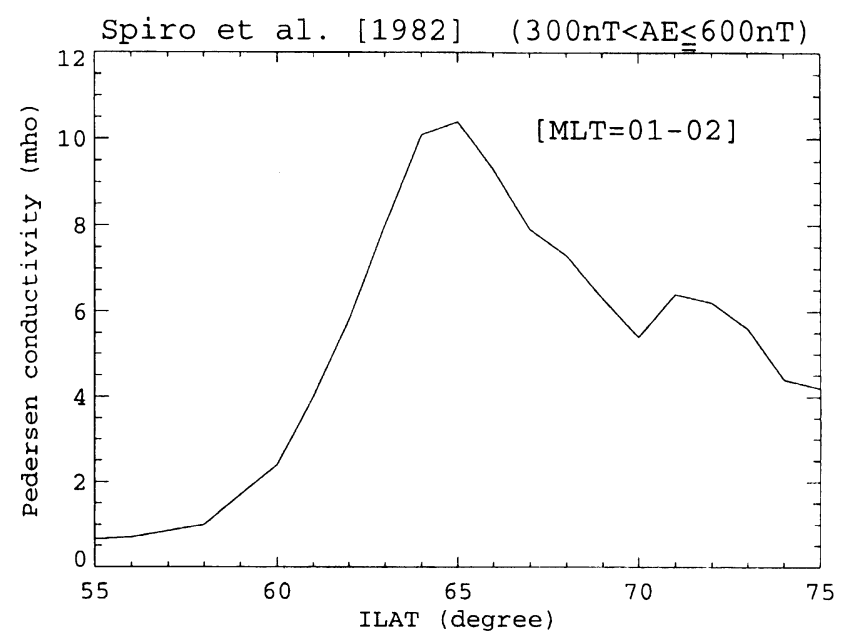

Fig. 9. ILAT dependence of the height-integrated Pedersen conductivity by Spiro et al. (1982) in the postmidnight sector (01-02MLT) for $300 \mathrm{nT}<\mathrm{AE} \leq 600 \mathrm{nT}$ condition. 
Table 3. Dependence of the duration of the pulsation $\tau$ on the ionospheric Pedersen conductivity $\Sigma_{P}$.

\begin{tabular}{lccccccc}
\hline$\Sigma_{P}$ (mho) & 4 & 6 & 8 & 9 & 10 & 11 & 12 \\
\hline$\tau\left(=\gamma^{-1}\right)$ (seconds) & 159 & 239 & 320 & 360 & 400 & 440 & 480 \\
\hline
\end{tabular}

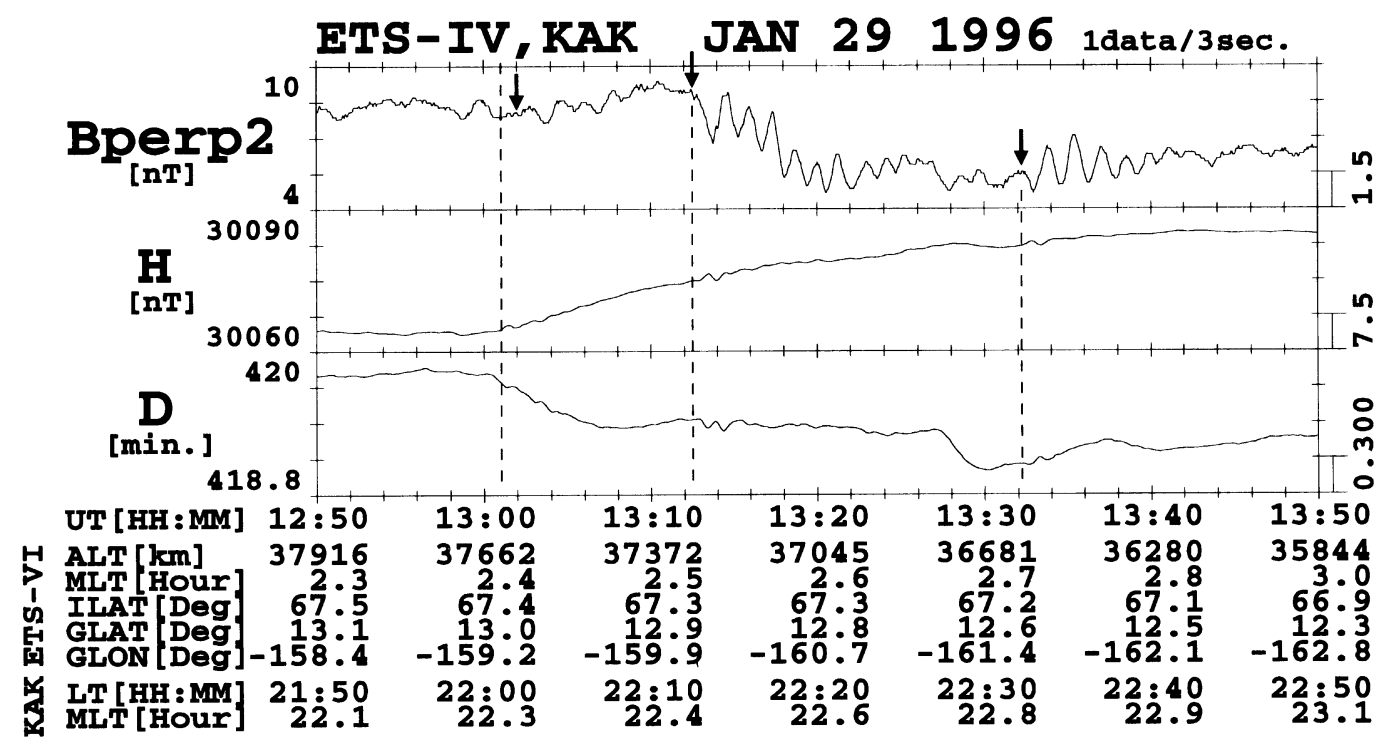

Fig. 10. The first panel gives the magnetic field variation in the azimuthal component from ETS-VI, which is the same event as that of Fig. 5. The second and third panels show the $H$ - and $D$-components of the geomagnetic field at Kakioka. Three nightside Pc 4 pulsations in the magnetosphere appeared from 1302:00UT, 1312:30UT, and 1332:30UT, which are indicated by arrows. The vertical broken lines show onset times of Pi 2 pulsation observed on the ground (1301:00UT, 1312:30UT, and 1332:30UT). The onset of the nightside Pc 4 pulsation is simultaneous to or one minute later than that of Pi 2 pulsation.

source for the nightside Pc 4 pulsations, because substorms are distinctive phenomena occurring on the nightside. The above calculation that even pulsations which are excited by an impulsive source can be sustained for a little longer than 67 minutes also supports our expectation. To test the validity of our expectation, we utilized the magnetic field data from the Kakioka (KAK) ground observatory $\left(26.9^{\circ}\right.$ geomagnetic latitude, $\mathrm{LT}=\mathrm{UT}+9)$.

The first panel of Fig. 10 gives the magnetic field variation in the azimuthal component observed by ETS-VI, which is the same event as that of Fig. 5, and the second and third panels show the $H$ - and $D$-components of the geomagnetic field at Kakioka. ETS-VI and Kakioka were around 02-03MLT and 22-23MLT, respectively. In the first panel three nightside Pc 4 pulsations in the magnetosphere appeared from 1302:00UT, 1312:30UT, and 1332:30UT which are indicated by arrows. These waves had continuous waveforms with periods of 80-100 seconds. The vertical broken lines show onset time of waves observed on the ground (1301:00UT, 1312:30UT, and 1332:30UT). We notice that these waves had periods of about 60 seconds and were damped within a few cycles, though the nightside Pc 4 pusations observed by the ETS-VI satellite lasted for about 10 minutes. Thus we consider these waves to be low-latitude Pi 2 pulsations, which are closely related to magnetospheric substorms (e.g., Saito et al., 1976; Sakurai and Saito, 1976). Comparing the arrows in the first panel with the vertical lines, we see that the onset of the nightside $\mathrm{Pc} 4$ pulsation is simultaneous to or one minute later than that of the low-latitude Pi 2 pulsation. This fact shows that the nightside Pc 4 pulsations occur at almost the same time of magnetospheric substorms.

Figure 11 shows another event of nightside Pc 4 pulsation and low-latitude Pi 2 pulsation, which appeared on 27 December 1994, in the same format as Fig. 10. A nightside Pc 4 pulsation started at 1054:00UT and continued to oscillate for about 15 minutes. At Kakioka a low-latitude Pi 2 pulsation whose onset time was 1053:00UT was observed around 20.3MLT. Duration of the Pi 2 pulsation is much shorter than that of the nightside Pc 4 pulsation. There is the time difference of one minute between the onset of the nightside Pc 4 pulsation and that of the Pi 2 pulsation. Figures 10 and 11 lead us to conclude that the nightside Pc 4 pulsations are associated with magnetospheric substorms.

We investigated difference of the onset time statistically, which is defined as the onset time of the nightside Pc 4 pulsation minus that of the low-latitude Pi 2 pulsation. Only the events in which Kakioka observed low-latitude Pi 2 pulsations in the MLT range of 19-01MLT were used. The difference of the onset time is plotted against MLT in which ETS-VI observed nightside Pc 4 pulsation (Fig. 12). A bigger circle in Fig. 12 represents two events. The time difference is zero or positive for almost all the events. The result indicates 


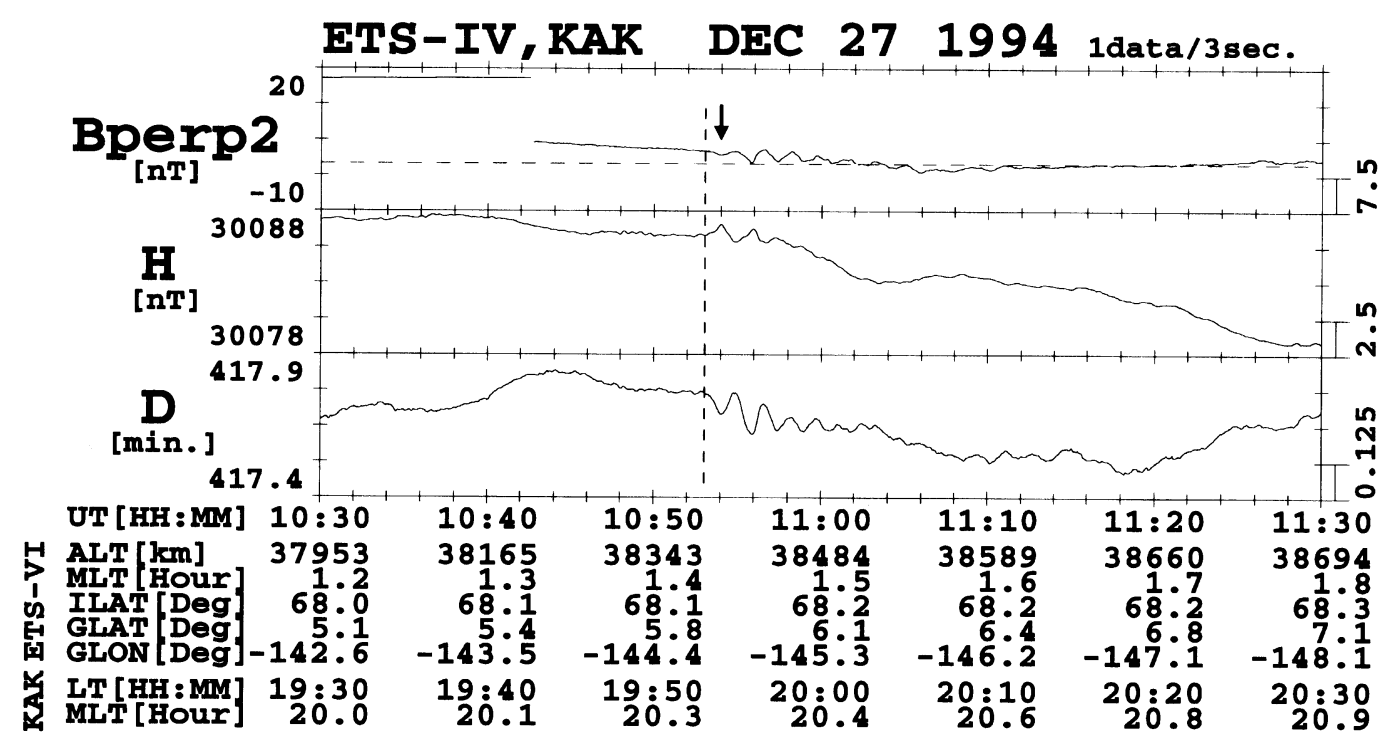

Fig. 11. A nightside Pc 4 pulsation observed by ETS-VI and a Pi 2 pulsation observed at Kakioka which appeared on 27 December 1994 . The nightside Pc 4 pulsation started at 1054:00UT (an arrow), and the Pi 2 pulsation started at 1053:00UT (a vertical broken line). The difference of onset time between Pc 4 and Pi 2 is one minute. Format is same as for Fig. 10.

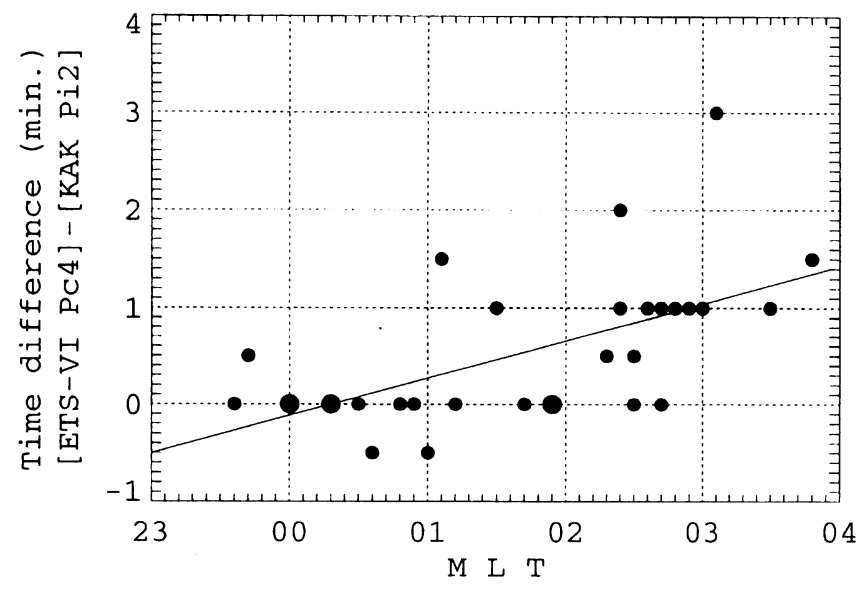

Fig. 12. MLT dependence of the onset time difference which is defined as the onset time of the nightside Pc 4 pulsation minus that of the low-latitude Pi 2 pulsation. A bigger circle represents two events. A solid line represents a regression line.

that the nightside Pc 4 pulsations appear at the same time of or a few minutes later than the low-latitude Pi 2 pulsations, which is consistent with Figs. 10 and 11 . We notice that the difference of the onset time tends to become larger for ETS-VI satellite locations further from the midnight. This shows that the sources for Pc 4 excitation are released at the same time of substorm onset and propagate from midnight to dawn. Drawing a regression line in Fig. 12, we found that the sources propagated at the velocity of about 45 degrees per 1.2 minutes $\left(1.1 \times 10^{-2} \mathrm{rad} / \mathrm{s}\right)$.

Two possible scenarios are considered for excitation mechanisms of the nightside Pc 4 pulsations. (1) A fast mode wave radiated at substorm onset will excite azimuthally polarized waves at a local magnetic field line, whose eigenfrequency is covered by the spectrum of the fast mode wave. This scenario is similar to the so-called field line resonance model to explain Pc 5 pulsations on the dayside (Southwood, 1974; Chen and Hasegawa, 1974). (2) Energetic electrons injected at substorm onset drift eastward and might excite azimuthally polarized waves through an unspecified wave-particle interaction. We estimated the propagation velocity of sources for both scenarios and compared them with the observed delay times. We assume that the sources for Pc 4 excitation, that is, fast mode Alfvén waves or energetic electrons, are released at 22MLT, around where the occurrence probability of Pi 2 pulsation shows a peak (Fig. 2 of Singer et al. (1983)). In the case of the first scenario, we calculated the propagation time from a region where the substorm occurred $(L=20)$ to the location of the ETS-VI satellite $(L=7)$ as shown in Fig. 13, and derived the azimuthal propagation velocity to be $2.1 \times 10^{-2}-2.8 \times 10^{-2} \mathrm{rad} / \mathrm{s}$. Even if we assume that the substorm occurred at a region closer to the earth $(L=15,10$, and $8)$, the estimated azimuthal propagation velocity $\left(2.3 \times 10^{-2}-\right.$ $3.0 \times 10^{-2} \mathrm{rad} / \mathrm{s}, 2.5 \times 10^{-2}-4.0 \times 10^{-2} \mathrm{rad} / \mathrm{s}$, and $2.7 \times 10^{-2}-$ $4.8 \times 10^{-2} \mathrm{rad} / \mathrm{s}$, respectively) is not so different from that for $L=20$. In the second scenario, the energetic electrons were assumed to be injected to the region around $L=7$ and drift eastward. In the dipole magnetic field, the particle's bounce averaged drift velocity $\omega_{d}$ is given as follows (Hamlin et al., 1961):

$$
\omega_{d} \sim \frac{3 v^{2}}{\Omega_{c}\left(R_{E} L\right)^{2}}(0.35+0.15 \sin \alpha)
$$

where $v$ is particle velocity, $\Omega_{c}$ is the gyrofrequency, $R_{E}$ is the Earth's radius, and $\alpha$ is the equatorial pitch angle. If we take electrons with energy of 3-30 keV and pitch angle of $90^{\circ}$ at $L=7$, we obtain $\omega_{d} \sim 5.1 \times 10^{-5}-5.1 \times 10^{-4} \mathrm{rad} / \mathrm{s}$. The estimation for Alfvén wave velocity are consistent with the observational result, but the estimated drift velocity of 


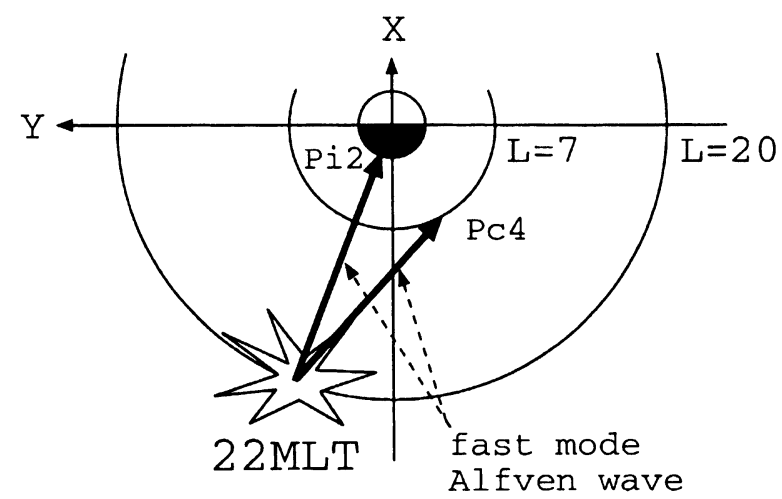

Fig. 13. A schematic figure to estimate the azimuthal propagation velocity of fast mode Alfvén wave. It was assumed that substorm occurred in the region of 22MLT at $L=20$.

energetic electrons is much smaller than the observed. We therefore adopt the first scenario for excitation mechanism of the nightside Pc 4 pulsations.

Figure 12 shows that events in which the difference of the onset time is zero were found in the MLT range from 23.6MLT through midnight to 2.7MLT. This means that the propagation time of fast mode Alfvén wave from substorm occurrence region to the location of ETS-VI in the MLT range of 23.6-2.7MLT is the same as that from substorm occurrence region to the ground (Fig. 13). From the calculation of the propagation time, we found that the fast mode Alfvén wave can propagate to the region of $1.4 \mathrm{MLT}$ at $L=7$ while fast mode Alfvén wave propagate from substorm occurrence region to the ground. The MLT value of $1.4 \mathrm{MLT}$ is contained in the observed MLT range of 23.6-2.7MLT. This result supports the first scenario that fast mode Alfvén waves excite the nightside Pc 4 pulsations. The spread of the observed MLT range of 23.6-2.7MLT is thought to be caused by change of the location where substorm occurred.

4.45 Comparison with the previous studies Figure 6 indicates that the nightside Pc 4 pulsations occur more frequently in the postmidnight sector (23-04MLT) than in the premidnight sector (19-23MLT). The occurrence probability of the nightside Pc 4 pulsations has a minimum around 22-23MLT consistent with the result of Saka et al. (1996a). Thus we can say that the occurrence of the nightside Pc 4 pulsations has an asymmetry to the substorm occurrence region. Takahashi et al. (1996) found a similar asymmetry in occurrence of TTW, and considered that masking effects due to nonsinusoidal magnetic fluctuations following substorms are more prominent in the premidnight sector. We suggest that the asymmetry in nightside Pc 4 occurrence might be explained by an asymmetry in the ionospheric Pedersen conductivity. Figure 14 shows MLT dependence of the average ionospheric Pedersen conductivity in the region of $64^{\circ}-$ $68^{\circ}$ ILAT for $300 \mathrm{nT}<\mathrm{AE} \leq 600 \mathrm{nT}$ condition, which was derived from Table A2 of Spiro et al. (1982). The Pedersen conductivity for 23-04MLT (8.5-9.5 mho) is higher than that for 19-23MLT (4.5-8.0 mho). As we can see in Table 3, the smaller the ionospheric Pedersen conductivity, the shorter the duration of pulsations. Therefore pulsations in the premid-

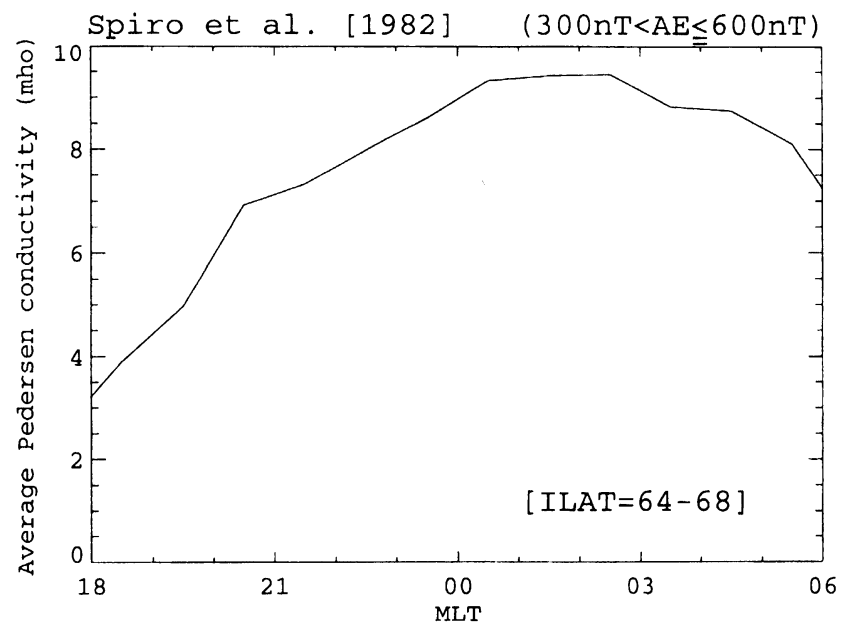

Fig. 14. MLT dependence of the average ionospheric Pedersen conductivity in the region of $64^{\circ}-68^{\circ} \mathrm{ILAT}$ for $300 \mathrm{nT}<\mathrm{AE} \leq 600 \mathrm{nT}$ condition, which was derived from Table A2 of Spiro et al. (1982).

night sector (19-23MLT) are damped more quickly and less readily observed than those in the postmidnight sector (2304MLT). We consider that irregular magnetic disturbances accompanying substorm onsets are localized around the substorm occurrence region, and these disturbances cause the smallest occurrence probability of Pc 4 around 22-23MLT by masking or changing continuous waveforms of the nightside Pc 4 pulsations.

We concluded that the nightside Pc 4 pulsations are the fundamental mode of standing Alfvén waves. This is consistent with the results by Takahashi et al. (1996) that TTWs are standing Alfvén waves on individual magnetic field lines, which are suggested from the TTW frequencies at two satellites (AMPTE/CCE and GOES-5) and the phase relationship between oscillations in magnetic field and ion flux anisotropy.

Takahashi et al. (1996) solved the axisymmetric toroidal mode equation with the ionospheric conductivity model and concluded that a TTW lasts for about 5 cycles for $L \sim 7$. If we assume the period of pulsations of $80-100$ seconds, we found that a TTW lasts for 400-500 seconds. This duration is consistent with our calculation of 6-7 minutes.

Takahashi et al. (1996) proposed a change in magnetospheric convection during substorms for energy sources for TTW. The change of magnetospheric convection is transmitted to the ionosphere via Alfvén waves, and leads to damped transient standing Alfvén waves. They insisted that TTW is excited by even a small substorm or pseudo substorm which could change the convection pattern. Saka et al. (1996a, b) presented a model in which plasma pressure generated in the nightside magnetosphere in association with energetic particle injections acts as an in situ sources of QPO. They showed an azimuthal pressure gradient provided by energetic particle injections increases the field line curvature and leads to transient field line oscillations. Using the electron flux data for the energy ranges 30-300 keV from the geosynchronous satellites, Saka et al. (1996b) demonstrated that QPO observed by GOES-5 diminished when the pressure gradient was reduced. From a viewpoint of propagation velocity of 
sources, we consider that the nightside Pc 4 pulsations are excited through coupling to the fast mode Alfvén waves which are radiated at substorm onsets, rather than excited by energetic electrons injected at substorm onset. Even if we take high-energy electrons $(30-300 \mathrm{keV})$, the estimated drift velocity of these electrons $\left(5.1 \times 10^{-4}-5.1 \times 10^{-3} \mathrm{rad} / \mathrm{s}\right)$ is still smaller than the observation. The fast mode Alfvén waves are thought to be radiated at even a pseudo substorm onset, because Fig. 6(b) of Takahashi et al. (1996) showed that a Pi 2 pulsation appeared in association with a pseudo substorm. Thus the excitation mechanism of the nightside Pc 4 pulsations proposed in this study is available in both cases of substorms and pseudo substorms.

The excitation mechanism proposed above is supported by the first and second events in Fig. 5, in which short-lived disturbances in the compressional $\left(\Delta B_{\|}\right)$component appeared to be coincident with the nightside Pc 4 pulsations. These compressional disturbances are thought to be the fast mode Alfvén waves which were launched at substorm onsets and excited the nightside Pc 4 pulsations. It should be noted that the amplitudes of these compressional disturbances were very small $(0.4 \mathrm{nT}$ and $0.8 \mathrm{nT}$ for the first and second events, respectively). In the third event in Fig. 5, however, there was no clear disturbances in the compressional component at 1332UT when the nightside Pc 4 pulsation started. We can expect that the magnitude of substorm in the third event was smaller than that in the second event, because amplitude of the ground Pi 2 pulsation in the third event was smaller than that in the second event (Fig. 10). Thus one of the possible interpretations of this event is that the amplitude of compressional disturbances was so small that the magnetometer on board ETS-VI could not detect the disturbance (Note that the resolution in Range-H was 0.125 nT.). In the another interpretation, this event might be explained by the model proposed by Takahashi et al. (1996) because their model needs no consistent compressional disturbances at the onset of TTW. Although the propagation velocity of magnetospheric convection change is not clear, it might be as large as that of fast mode Alfvén waves. Further studies will be necessary to conclude which one of the three mechanisms is the most plausible.

A few previous studies have reported azimuthally polarized Pi 2 pulsations observed by satellites. Pi 2 pulsation which was dominant in the east-west component was observed by ATS-6 around 02MLT (Kuwashima, 1983). Sakurai and McPherron (1983) found Pi 2 pulsation with an obvious sinusoidal oscillation only in the east-west component which were observed by ATS-6 around 02LT. Though these pulsations were called as Pi 2 pulsation, they should be classified into the nightside Pc 4 pulsations because they have continuous waveforms which are dominant in the azimuthal component and were observed around 02MLT where we found an occurrence peak of the nightside Pc 4 pulsations.

\section{Conclusions}

The magnetic field data from the ETS-VI satellite were analyzed statistically to investigate magnetic pulsations in Pc 3-5 bands. We found prominent features in the occurrence distributions of the four pulsations: (1) azimuthal Pc 5 pulsation; (2) azimuthal Pc 3 pulsation; (3) radial Pc 4 pul- sation on the dayside; (4) azimuthal Pc 4 pulsation on the nightside. The main results for these pulsations are as follows.

1. The azimuthal Pc 5 pulsations occur frequently in the MLT ranges of both 05-09MLT and 15-18MLT, and are observed mostly in the off equatorial region. They are considered as the fundamental mode of the standing Alfvén waves. The energy of the azimuthal Pc 5 pulsations is thought to be supplied by the Kelvin-Helmholtz instability on the magnetopause.

2. The azimuthal Pc 3 pulsations appear frequently in the wide MLT range of $07-16$ MLT. The azimuthal Pc 3 pulsations are thought to be higher harmonics of the standing Alfvén waves, which can explain the observation that the frequencies of Pc 3 pulsations become lower as the satellite moves away from the earth.

3. The radial Pc 4 pulsations have high occurrence on the afternoon side (11-20MLT) and an occurrence peak in the MLT-ILAT area of $14-16 \mathrm{MLT}$ and $66^{\circ}-67.5^{\circ} \mathrm{ILAT}$. They might be excited by bounce resonance, as suggested by the previous studies (Arthur and McPherron, 1981; Takahashi and McPherron, 1984; Anderson et al., 1990a, Takahashi and Anderson, 1992).

4. We have investigated in detail the azimuthal Pc 4 pulsations on the nightside (nightside Pc 4 pulsations), which were called as TTW by Takahashi et al. (1996) and as QPO by Saka et al. (1996a, b), and obtained the following results. (a) The nightside Pc 4 pulsations have high occurrence in the MLT range of 23-04MLT and an occurrence peak at 0102MLT.

(b) They are considered as the fundamental mode of the standing Alfvén waves. This is supported by correspondence between the eigenperiod calculated from standing wave model and the observed period, and the observation that the nightside Pc 4 pulsations are less observed near the geomagnetic equator.

(c) The nightside Pc 4 pulsations continue to oscillate for about 10 minutes, which are expected from calculation of damping rate of standing waves on the nightside.

(d) The nightside Pc 4 pulsations start at almost the same time of magnetospheric substorm onsets, but they are different from Pi 2 pulsations in the magnetosphere. They are thought to be excited through coupling to the fast mode Alfvén waves launched at substorm onsets.

The excitation mechanism(s) for the radial Pc 4 pulsations on the dayside was left for future study. In respect of the nightside Pc 4 pulsations, we have proposed an excitation mechanism which is different from the mechanism proposed by Takahashi et al. (1996) and that by Saka et al. (1996a, b). It will be necessary to examine by further studies which one of the three excitation mechanisms is the most plausible. A simultaneous observation by satellites and ground stations which are on the same geomagnetic field line will be important to investigate the nightside Pc 4 pulsations.

Acknowledgments. We thank K. Takahashi and O. Saka for their valuable comments. We also thank T. Kamei for his technical support with the computer system. This study has been supported in part by grants from JSPS Research Fellowships for Young Scientists. 


\section{References}

Anderson, B. J., M. J. Engebretson, S. P. Rounds, L. J. Zanetti, and T. A. Potemra, A statistical study of Pc 3-5 pulsations observed by the AMPTE/CCE magnetic fields experiment, 1. Occurrence distributions, J. Geophys. Res., 95, 10495-10523, 1990a.

Anderson, B. J., T. A. Potemra, L. J. Zanetti, and M. J. Engebretson, Statistical correlations between Pc 3-5 pulsations and solar wind/IMF parameters and geomagnetic indicies, Physics of Space Plasmas, SPI Conference Proceedings and Reprint Series, Number 10, pp. 419-429, Scientific Publishers, Inc., Cambridge, MA, 1990 b.

Arthur, C. W. and R. L. McPherron, Micropulsations in the morning sector, 3. Simultaneous ground-satellite observations of 10 - to 45 -s period waves near $L=6.6, J$. Geophys. Res., 82, 2859-2866, 1977.

Arthur, C. W. and R. L. McPherron, The statistical character of Pc 4 magnetic pulsations at synchronous orbit, J. Geophys. Res., 86, 1325-1334, 1981.

Chapell, C. R., K. K. Harris, and G. W. Sharp, A study of the influence of magnetic activity on the location of the plasmapause as measured by OGO 5, J. Geophys. Res., 75, 50-56, 1970.

Chen, L. and A. Hasegawa, A theory of long-period magnetic pulsations, 1. Steady state excitation of field line resonance, J. Geophys. Res., 79, 1024-1037, 1974

Cummings, W. D., R. J. O’Sullivan, and P. J. Coleman, Jr., Standing Alfvén waves in the magnetosphere, J. Geophys. Res., 74, 778-793, 1969.

Ellis, P. and D. J. Southwood, Reflection of Alfvén waves by non-uniform ionospheres, Planet. Space Sci., 31, 107-117, 1983.

Engebretson, M. J., L. J. Zanetti, T. A. Potemra, and M. H. Acuna, Harmonically structured ULF pulsations observed by the AMPTE CCE magnetic field experiment, Geophys. Res. Lett., 13, 905-908, 1986.

Hamlin, D. A., R. Karplus, R. C. Vik, and K. M. Watson, Mirror and azimuthal drift frequencies for geomagnetically trapped particles, J. Geophys. Res., 66, 1-4, 1961.

Hughes, W. J., Magnetospheric ULF waves: A tutorial with a historical perspective, in Solar Wind Sources of Magnetospheric Ultra-Low-Frequency Waves, Geophys. Monogr. Ser, vol. 81, edited by M. J. Engebretson, K. Takahashi, and M. Scholer, pp. 1-11, AGU, Washington, D. C., 1994.

Jacobs, J. A., Y. Kato, S. Matsushita, and V. A. Troitskaya, Classification of geomagnetic micropulsations, J. Geophys. Res., 69, 180-181, 1964.

Kokubun, S., Statistical characteristics of Pc 5 waves at geostationary orbit, J. Geomag. Geoelectr., 37, 759-779, 1985.

Kokubun, S., K. N. Erickson, T. A. Fritz, and R. L. McPherron, Local time asymmetry of Pc 4-5 pulsations and associated particle modulations at synchronous orbit, J. Geophys. Res., 94, 6607-6625, 1989.

Kuwashima, M., Wave characteristics of magnetic Pi 2 pulsations in the magnetosphere and on the ground, Mem. Kakioka Magn. Obs., 20, 1-15, 1983.

Lin, C. C. and L. J. Cahill, Jr., Pi 2 pulsations in the magnetosphere, Planet. Space Sci., 23, 693-711, 1975.

Nagai, T., T. Ondoh, H. Matsumoto, T. Goka, T. Fukuda, M. Nosé, T. Iyemori, K. Takahashi, and S. Kokubun, ETS-VI magnetic field observations of the near-earth magnetotail during substorms, J. Geomag. Geoelectr.,
48, 741-748, 1996.

Nosé, M., T. Iyemori, M. Sugiura, and J. A. Slavin, A strong dawn/dusk asymmetry in Pc 5 pulsation occurrence observed by the DE-1 satellite, Geophys. Res. Lett., 22, 2053-2056, 1995.

Olbert, S., Summary of experimental results from M.I.T. detector on IMP1, in Physics of the Magnetosphere, edited by R. L. Carovillano, J. F. McClay, and H. R. Radoski, pp. 641-659, Reidel Doredrecht, 1968.

Potemra, T. A. and L. G. Blomberg, A survey of Pc 5 pulsations in the dayside high-latitude regions observed by Viking, J. Geophys. Res., 101, 24801-24813, 1996.

Saito, T., Long-period irregular magnetic pulsation, Pi 3, Space Sci. Rev., 21, 427-467, 1978

Saito, T., K. Yumoto, and Y. Koyama, Magnetic pulsation Pi 2 as a sensitive indicator of magnetospheric substorm, Planet. Space Sci., 24, 1025-1029, 1976.

Saka, O., H. Akaki, O. Watanabe, and D. N. Baker, Ground-satellite correlation of low-latitude Pi 2 pulsations: A quasi-periodic field line oscillation in the magnetosphere, J. Geophys. Res., 101, 15433-15440, 1996a.

Saka, O., O. Watanabe, and D. N. Baker, A possible driving source for transient field line oscillations in the postmidnight sector at geosynchronous altitudes, J. Geophys. Res., 101, 24719-24726, 1996 b.

Sakurai, T. and R. L. McPherron, Satellite observations of Pi 2 activity at synchronous orbit, J. Geophys. Res., 88, 7015-7027, 1983.

Sakurai, T. and T. Saito, Magnetic pulsation Pi 2 and substorm onset, Planet Space Sci., 24, 573-575, 1976.

Singer, H. J., W. J. Hughes, P. F. Fougere, and D. J. Knecht, The localization of Pi 2 pulsations: Ground-satellite observations, J. Geophys. Res., 88, 7029-7036, 1983

Snyder, C. W., M. Neugebauer, and U. R. Rao, The solar wind velocity and its correlation with cosmic-ray variations and with solar and geomagnetic activity, J. Geophys. Res., 68, 6361-6370, 1963.

Southwood, D. J., Some features of field line resonances in the magnetosphere, Planet. Space Sci., 22, 483-491, 1974.

Spiro, R. W., P. H. Reiff, and L. J. Maher, Jr., Precipitating electron energy flux and auroral zone conductances-An empirical model, J. Geophys. Res., 87, 8215-8227, 1982.

Takahashi, K. and B. J. Anderson, Distribution of ULF energy ( $f<80$ $\mathrm{mHz}$ ) in the inner magnetosphere: A statistical analysis of AMPTE CCE magnetic field data, J. Geophys. Res., 97, 10751-10773, 1992.

Takahashi, K. and R. L. McPherron, Standing hydromagnetic oscillations in the magnetosphere, Planet. Space Sci., 32, 1343-1359, 1984

Takahashi, K., S. Ohtani, and B. J. Anderson, Statistical analysis of Pi 2 pulsations observed by the AMPTE CCE spacecraft in the inner magnetosphere, J. Geophys. Res., 100, 21929-21941, 1995.

Takahashi, K., B. J. Anderson, and S. Ohtani, Multisatellite study of nightside transient toroidal waves, J. Geophys. Res., 101, 24815-24825, 1996.

M. Nosé (e-mail: nose@kugi.kyoto-u.ac.jp), T. Iyemori, S. Nakabe, T. Nagai, H. Matsumoto, and T. Goka 